\title{
Zur Infektionsverhütung im Säuglingsspital ${ }^{1}$ ). \\ Von

\author{
Privatdozent Ludwig F. Meyer.
}

(Aus dem Waisenhause der Stadt Berlin [Oberarzt: Prof. Dr. Finkelstein].)

Mit 16 Textfignren.

Als vor anderthalb Jahrzehnten Heubners Studie über „Säuylingsernährung und Säuglingsspitäler" erschien, befand sich die Säuglingsverpflegung in geschlossener Anstalt noch in den ersten, nicht gerade glücklichen Anfängen. Heubner wies damals klar den Weg, der zur Erreichung befriedigender Resultate bei der Anstaltspflege einzuschlagen war. Auf Grund der von ihm und seinem damaligen Assistenten Finkelstein gesammelten Erfahrungen vertrat Heubner den Standpunkt, daß Infektionen, vorwiegend vom Magendarmkanal aus, die Ernährungserfolge in der Anstalt vereitelten. Es waren, so schrieb Heubner damals, ,die kleinen Infektionen, oder sagen wir, um alles Hypothetische zu vermeiden, die kleinen Erkrankungen, die jeden vorübergehenden Erfolg immer wieder zum Scheitern bringen und deren immer neue Wiederkehr schließlich das verhängnisvolle Ziel erreicht". Diese Auffassung des „Hospitalismus“ führte zu einer Reform der Säuglingspflege in Deutschland. Sie schuf die aseptisch isolierende Pflege des Säuglings.

Was durch die Asepsis im Säuglingsspital, die nun Hand in Hand ging mit der Vervollkommnung der Pflegeleistung und der Ernährungstechnik, erreicht worden ist, bedarf an dieser Stelle keiner Schilderung. Die Erfolge der Anstaltsbehandlung kranker Säuglinge geben allenthalben davon Kunde. Trotzdem gibt wohl jeder Anstaltsarzt zu, daß die Erfolge auch heutzutage das Optimum noch nicht erreichen. Ein kleiner Rest des „Hospitalismus", über dessen Natur man verschiedener Meinung ist, wird allgemein dafür verantwortlich gemacht. Dic cineu führen ihn auf Unvollkommenheiten in der Pflege, die andern auf solche der Ernährungstechnilk, wieder andere auf Schäden des ,Milicus ${ }^{6}$ zurück. Ein treffliches Spiegelbild der verschiedenen Auffassungen hat erst jüngst Freund ${ }^{2}$ ) gegeben.

1) Erschien gleichzeitig in der Festschrift für Hcu bner.

${ }^{2}$ ) Uber den „Hospitalismus“ der Säuglinge. Ergebnisse d. imm. Medizin u. Kinderheilk. B. VI, S. 133. 
Beobachtungen, die ich im Kinderasyl und Waisenhaus der Stadt Berlin in dieser Richtung erneut angestellt habe ${ }^{1}$ ), schienen darauf hinzuweisen, daß wenigstens für unsere Anstalten immer noch die Infektion, und zwar in erster Linie und fast ausschließlich die Grippe im Spital die Hauptsehuld daran trägt, wenn in der Anstalt ein Idealerfolg nicht erzielt wird. Nicht nur, daß die Erkrankungen der Luftwege einen größeren Prozentsatz der Todesfälle ausmachten als die Ernährungsstörungen (im Jahre 1910/11 12,2\% der Todesfälle an Lrnährungsstörungen, $35 \%$ an Respirationserkrankungen), und von 3000 Säuglingen im Jahre $1910 / 1160$ d. s. $2 \%$ dahinrafften, kein Kind, das länger als 4 Wochen in der Austalt verweilte, blieb nach diesen Feststellungen von der Erkrankung der Luftwege verschont. Die enorme Häufigkeit der grippalen Erkrankungen im Spital gibt ein Quotient wieder, dessen Zähler die Summe der Infektionen und dessen Nenner clie Zahl der Kinder mit einer bestimmten Aufenthaltsdauer darstellt. Danach erleidet ein Säugling bei einem Anstaltsaufenthalt

$$
\begin{aligned}
& \text { von 7- 14 Tagen 0,25 Infektionen der Atmungsorgane } \\
& \text {, } 14-30 \quad \text { " } 0,71 \quad \text { " " " } \\
& \text {., } 30-60 \quad \text {, } 1,3 \quad \text { " } \quad \text { ", } \\
& \text {., } 60-80 \quad \text { " } 2,82 \quad \text {, " , } \\
& \text { " } 80-100 \quad, \quad 3,08 \quad, \quad, \quad,
\end{aligned}
$$

Mit anderen Worten heißt das: Ein Kind, das sich länger als einen Monat im Spital aufhält, hat die Aussicht, durchschnittlich eine Affektion der Luftwege zu erwerben. Bei einem Spitalsaufenthalt von über 100 Tage macht ein Individuum durchschnittlich 3 solcher Infektionen durch.

Die Bedeutung der grippalen Infektion geht aber weit über die einer Lokalerkrankung der Luftwege hinaus. Wie ich in der vorher zitierten Arbeit durch klinisch-experimentelle Untersuchungen erneut vor Augen führte, ist der Infekt imstande, jede Form der Ernährungsstörung auszulösen (vgl. auch $\mathrm{Risel}^{2}$ ) und Schlo $\left.ß^{3}\right)$ ). Man mußte daher die Frage aufwerfen, ob die Beziehungen zwischen Infektion und Emährungsvorgang nicht den Schlüssel zum Verständnis des milden Hospitalismus von heute liefern?

1) Uber den Hospitalismus der Säuglinge, Verlag von s. Karger 1913.

2) Uber Grippe im Kindesalter. Ergebnisse f. inn. Medizin u. Kinderheilk. B. VIII, s. 211 .

3) Über Säuglingsernährung. Verlag von S. Karger 1912. 
Bejaht man cliese Frage, wie wir es getan haben, dann wird man eine Hauptaufgabe des Anstaltsarztes darin erblicken müssen, die Einschleppung der Infektionen ins Spital einzuschränken.

Dieses Problem kann von drei verschiedenen Seiten angefaßt werden:

1. Dureh Verbesserung der Frnährungsbedingungen. Wie zur Genüge feststcht, ist die Krankheitsbereitschaft am geringsten bei natürlich ernährten Säuglingen. Je nnehr also mit Fraucnmileh ernährt wird, desto geringer wird die Zahl der Infizierten sein.

2. Dureh Verbesserung der Pflege. Die hohe Bedeutung, die die minutiös arbeitende Versorgung des Kinindes für dessen Wohlbefinden besitzt, steht außer Frage. Und da Wohlbefinden und Krankheitsbereitschaft ungekclirt proportional sind, dürfte die Verpflegungswoise, whe daßs man es weiter definieren kam, bei der Bekämpfung der Infektion von nicht zu unterschätzender Bedeutung sein.

3. Durch direkte Verhinderụng der Infektionsübertragung. So einfach dieser letzte Weg zu sein scheint, so viel Hindernisse stellen sich ihm, wic jeder Anstaltsarzt weiß, in den Weg. Trotz dieser Schwiorigkeiten will ich in folgendem versuchen, diesen Weg zu gehen, da den beilen ersten Möglichkeiten im Rahmen eines kommunalen Anstaltsbetriebes aus leichtbegreiflichen materiellen Gründen eine Grenze gesetzt ist, die wir bereits orreicht haben.

Gelingt die Eindämmung der Erkrankungen der Luftwege, so mülbte (ler letzte Rest des Hospitalschadens verschwinden, der sich hauptsïchlich heute noch geltend macht, in den sehlechten Rosultaten künstlich crnährter Neugeborener (Schelble), in der Häufigkeit von Durchfällen (Szana), im schlechten Gedeihen der Säuglinge mit exsulativer Diathese (Keller, Freund). Damit hätte man gleichzeitig den Beweis in Händen, daß in der Tat der Hospitalismus in seiner heutigen Form als Folgeerscheinung der Infektion anzusprechen ist und es entfielen Theorien, wie die von Schloß ${ }^{1}$ ), die in etwas mystischer Weise für das schlechtere Gedeihen der Anstaltsinsassen „Milieuschädlen“" verantwortlich machen.

Die Bekümpfung der Infektion hat von der Erfahrung auszugehon, daß die Utbertragung der Erkrankung auf die gesunden Insassen der Anstalt von den neueingelieferten oder in der Station selbst an Grippe erkrankten Säuglingen ausgeht. Es gilt also, den Kontakt mit diesen zu vermeiden. Das mag dort leicht sein, wo täglich nur ein geringer

1) 1. c. 
Wechsel in der Belegung erfolgt und die Zahl der Aufnahmen Grippekranker eine sehr geringe ist. Allerdings stellen sich auch hier schon deshalb Schwierigkeiten entgegen, weil man die Erkrankung oft erst festzustellen imstande ist, nachdem der Patient 1-2 Tage auf der Station lag und bereits andere Kinder infiziert hat. Daß in unserer Anstalt dieser Weg bei der großen Anzahl katarrhalisch erkrankter neuaufgenommener Kinder überhaupt nicht gangbar ist, lehrt ein Utberblick über die grippekrank aufgenommenen Kinder in der Zeit vom November 1911 bis November 1912:

\begin{tabular}{|c|c|c|c|c|c|c|c|}
\hline \multicolumn{6}{|c|}{ Monat } & \multirow{2}{*}{$\begin{array}{c}\begin{array}{c}\text { Absolute Zahl } \\
\text { der } \\
\text { Erkrankungen }\end{array} \\
28\end{array}$} & $\begin{array}{l}\text { Prozentzatz der } \\
\text { Erkrankten zu den } \\
\text { Aufgenommenen }\end{array}$ \\
\hline Novembe & & 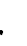 & . & - & & & $14,25 \%$ \\
\hline Dezember & & & . & . . & & 38 & $17,5 \%$ \\
\hline Januar & . & $\cdot$ & - & - . & ? & 28 & $12,9 \%$ \\
\hline Februar & . . & • & . & $=$ & . & 43 & $17,0 \%$ \\
\hline Mäxz . & - & . & . & . . & . & 37 & $13,25 \%$ \\
\hline April . & . . & . & . & . . & * & 40 & $16,5 \%$ \\
\hline Mai & . & . & . & . & & 34 & $12,5 \%$ \\
\hline Juni & • & & . & . & & 45 & $18,5 \%$ \\
\hline Juli . . & & & & · & & 34 & $10,75 \%$ \\
\hline August & & . & • & - & & 29 & $11,0 \%$ \\
\hline Septembe & $\mathrm{r}$. & . & . & . & & 32 & $15,0 \%$ \\
\hline Oktober & . & & . & . . & & 29 & $11,5 \%$ \\
\hline
\end{tabular}

Von 2964 Neueinlieferungen waren insgesamt 417 mit Erkrankungen der Luftwege behaftet.

Nicht uninteressant wäre es, der Frage nachzugehen, wieso sich unter den Neuaufgenommenen eine so große Anzahl Grippekranker findet. Die Säuglinge kommen meist nach längerem Transport in reduzierter Bekleidung aus Entbindungsanstalten oder aus der Familie in das Haus. Es ist daher nieht von der Hand zu weisen, daß äußere Schädlichkeiten, wie Abkühlung und Mangel jeder Verpflegung in Tagen oder Stunden vor der Aufnahme an dem Zustandekommen der grippalen Erkrankung beteiligt sind.

Der Aufgabe, 417 grippekranke Kinder vor einem Kontakt mit den anderen Anstaltsinsassen zu bewahren, sind wir nicht gerecht geworden. Nach genauen Aufzeichnungen wurden durch diese, sei es direkt, sei es indirekt durch Mittelspersonen, 815 Kinder mit Grippe infiziert. Mit anderen Worten: Jede Erkrankung der oberen Luftwege, 
die ins Haus eingeliefert wurde, hatte 2 weitere derartige Erkrankungen zur Folge.

Will man der Ausbreitung der Grippe mit Erfolg entgegentreten, so muß man vor allem den Weg der Infektion zu erkennen suchen. Uber ihn ist man bis heute noch getrennter Meinung. Lange Zeit hindurch nahm man ausschließlich eine indire k te Utbertragung der Grippe an. Als Vertreter dieser Anschauung schrieb Vog t ${ }^{1}$ ) noch jüngst: ,,Wenn die Betten genügend weit voneinander entfernt stehen $(1,50 \mathrm{~m})$ und sonstige Annäherung der Kinder etwả durch gleichzeitiges Tragen durch eine Person usw. vermieden wird, so können Respirationserkrankungen nur durch das Pflegepersonal übertragen werden. Der Transport versprühter Kieime durch den Luftstrom wird praktisch kaum ins Gewicht fallen."

Aber selbst unter den von Vogt gemachten Voraussetzungen dürfte bei aller Anerkennung des indirekten Uertragungsmodus eine solche Ablehnung der Ubertragung durch den Luftweg nach den ausgedehnten Untersuchungsreihen Flügges ${ }^{2}$ ) nicht berechtigt sein. „Ungleich größere Infektionsgefahr müssen“, so schrieb Flügge, „die in die Luft verschleuderten Tröpfchen bei den mit Husten und Auswurf verbundenen ansteckenden Krankheiten des Rachens und der Atmungsorgane bieten. Bei Diphtherie, Phthise, Influenza, Keuchhusten, Pneumonie wird der Utbergang von Erregern in die Luft in Tröpfchenform häufig vorkommen. Nach den oben aufgeführten Versuchen ist gar nicht daran zu zweifeln, daß jedes Husten, Niesen, Schreien solche Tröpfchen des Sputums und Mundsekrets in reichlicher Menge in die Luft überführt, daß diese sich weit im Raume verbreiten und lange in der Luft schweben."

Da minimale Luftströmungen von weniger als $1 \mathrm{~mm}$ in der Sekunde ${ }^{3}$ ) solehe Tröpfchen fortzubewegen und länger als 4 Stunden schwebend zu erhalten imstande sind, kam Flügge zu der Schlußfolgerung, daß die Inhalation verspritzter Sekrettröpfchen zweifellos ein häufiger und gefährlicher, vielleicht der gefährlichste Infektionsmodus ist.

Trotz der scharfen Beweisführung hat diese Flüggesche Auffassung auf die Durchführung der Isolierungsmaßnahmen im Spital kaum einen

1) Vogt, Zur Prophylaxe und Ernährungstherapie der Lungenerkrankungen im Kindesalter. Therap. Monatshefte 1912, Heft 8, S. 566.

2) Die Verbreitungsweisen und Bekämpfung der Tuberkulose, Leipzig 1908.

3) Bei der gewöhnlichen Erneuerung der Luft im Innern eines Wohnraumes mit geschlossenen Fenstern beträgt die Lufthewegung $1-2 \mathrm{~mm}$ pro Sekunde $=1-1^{1} /{ }_{2}$ fache Erneuerung der gesamten Zimmerluft innerhalb 1 Stunde. 
größeren Einfluß ausgeübt. Frst die unabhängig von Flügge in Frankreich auf eben derselben Anschauung basierende Theorie von Lesage ${ }^{1}$ ) scheint, wie jüngst die Diskussion im Anschluß an den Vortrag v. Pirquets in Münster lehrte, zu einer Revision der herrschenden Meinungen zu führen. Ebenso wie Flügge nimmt Lesage bekanntlich an, daß der Luftzug die Erreger der verschiedenen Erkrankungen in die Ferne trägt und so die Ansteckung vermittelt. In Konsequenz dieser Annahme legt er kontagiöse Erkrankungen in halboffene Boxen eines großen Saales, dessen Ventilation durch besondere Vorrichtungen äußerst sehwach gehalten wird. Man mag mit noch so großer Skepsis an die Theorien Lesages herangehen, die Tatsache dor günstigen Resultate steht fest, die er mit diesem System der Unterbringung Infektionskranker (mit Ausnahme von Varizellen, deren Erroger er als allzu leicht flüchtig bezeichnet) erzielt hat.

Es crsehien mir daher der Versuch angezeigt, mit einem auf FlüggeLesageschen Gedankengängen aufgebauten System der Verbreitung der Grippe im Spital entgegenzutreten.

Gloichsam als Tastversuch wurde daher Anfang November 1911 eine Station nach diesen Grundsätzen in einfachster Weise cingerichtct. Wenn ich mir schon heute erlaube, über die dabei gesammelten Erfahrungen zu berichten, so geschieht es nicht, weil die Einrichtungen schon heute als nachahmenswert gelten dürfen - das sind sie nicht sondern weil uns prinzipiell bewiesen scheint, daß die direkte Utbertragung der katarrhalischen Erkrankungen von Kind zu Kind auf diese Weise verhindert werden kann.

\section{Beschreibung der Stationseinrichtungen.}

Zwei Forlorungen mußten erfüllt werden: 1. die Dämpfung der Ventilation, 2. die individuelle Isolierung jeles Kindes. Die Hifüllung dieser Forderungen ist notwendig, damit einmal ausgeschleuderte Keime nicht rerschleppt werden, sondern sich inncrhalb, des für den Kranken bestimmten Raumes zu Boden setzen und so der Vernichtung anheirnfallen.

Die Dämpfung der Luftzuströmung wurcle nach dem Vorgange Lesages durch ständigen Verschluß der Fenster und das An-

1) Vgl. den Bericht E. Müllers, Jahrb. f. Kinderheilk. 74, 450 und Referat des ersten Pariser internationalen pädiatrischen Kongresses 1912. Jahrb. f. Kinderheilk. $76,582$.

2) Anstatt des teuren perforierten Glasfensters kann man nach Finkelsteins Vorschlag bei Doppelfenstern die Außenscheibe eines liensterquadrates 
bringen einer fein perforierten Glasscheibe ${ }^{2}$ ) an einem oder zwei Fensterquadraten erreicht. Zwecks noch intensiverer Abschwächung des Luftstroms passierte die Luft nach ihrem Einströmen durch das Fenster ein großes im Holzgestell aufgestelltes Gazefilter, das der Größe der Saalfenster entsprach.

In welch exquisiter Weise die einfache Mullage die Luftströmung verlangsamt, davon kann man sich sehr leicht überzeugen, wenn man eine kleine Kerzenflamme vor dem Mull und hinter dem Mull brennen läßt. Die vor dem Mull aufgestellte wird unruhig flackern, die hinter dem Mull ganz ruhig brennen. Exakter kann man durch anemometrische Messungen diesen besänftigenden Einfluß des Mulls auf die Luftbewegung feststellen. In einem unserer Versuche zeigte der Anemometer vor dem Mull 23 Umdrehungen in einer Minute an, hinter rem Mull nur 10.

Der Abzug der verbrauchten Luft geschah durch den gewöhnlichen Ventilationsschacht im Sommer in der Höhe der Decke, im Winter in der des Fußbodens.

Besonders wurde auf die Vermeidung von Zugluft beim Öffnen und Schließen der Zimmertüre geachtet. Die gegenüber dem Fenster liegende Tür wurde verschlossen und der Eingang nur durch cine seitliche Tür, die zunächst in einen als Baderaum dienenden Vorranm führt, gestattet. Außerdem wurde durch einen großen Wandschirm, dessen Höhe die Türöffnung überragt, ein Abschluß gegen allzuscharf eindringende Luftströme erreicht.

Gewiß ist die Frage berechtigt, ob nicht durch solche encrgische Maßnahmen der Luftzufluß allzusehr verringert wird und die Lufterneuerung leidet. Durch Bestimmung des Kohlensäuregehaltes in der Luft (rorgenommen durch das stältische Untersuchnngsamt) ${ }^{1}$ ) wurde festgestellt, daß die Luft der Station $0,9-1$ p. m. $\mathrm{CO}_{2}$ enthielt; das ist ein Gehalt, der zwar nicht als ideal zu bezeichnen ist, aber auch nicht: die Grenze des Zulässigen überschreitet. Nach Rubner2) ist eine Luft. erst schlecht und für einen beständigen Aufenthalt untauglich, wemn der $\mathrm{CO}_{2}$-Gehalt mehr als $1 \mathrm{p}$. m. beträgt. Die Lufterneuerung ging in $24 \mathrm{Stm}$ und die Innenseite eines schräg gegenüberliegenden Quadrates entfernen und zwischen beide eine perforierte Blechleiste einsetzen. Das Glas des Innenfensters wird durch eine einfache Mullage ersetzt. Der Luftstrom wird so gobrochen und in der gewünschten Weise abgeschwächt.

1) Herrn Dr. Seligman n vom städt. Untersuchungsamt sage ich für scine freundliche Unterstützung und nützlichen Ratschläge auch an dieser Stelle meinen Dank.

2) Lehrbuch der Hygiene S. 217. 
den 21,5 mal vor sich, auch dieser Wert bleibt hinter dem sonst erwünschten von 48 zurück. Eine häufigere Erneuerung müßte aber wenigstens bei den jetzigen Stand der Vorrichtungen - mit einer nicht erwünschten Beschleunigung der Luftbewegung einhergehen, so daß vorläufig davon Abstand genommen wurde.

In einer zweiten, später eingerichteten Station befand sich die Abzugsklappe leider nicht vis-a-vis dem Fenster, sondern, wie aus der beigefügten Abbildung hervorgeht, an der Seitenwand des Zimmers, nur ca. $2 \mathrm{~m}$ vom Fenster entfernt. Der Luftstrom verteilte sich daher nicht gleichmäßig durch den Raum, sondern floß nach seinem Eintritt durch die Fensteröffnung vorzüglich den beiden ersten Boxen zu. Hier ent-

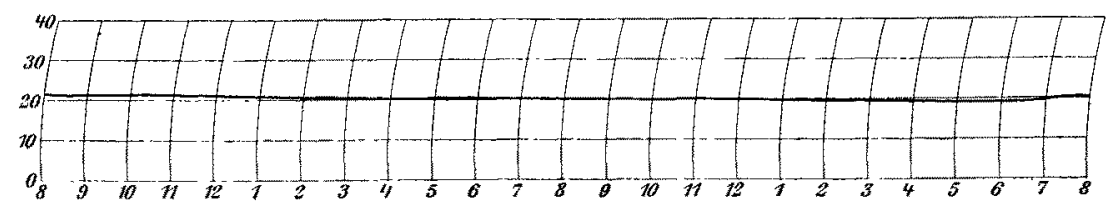

Temperatur der Boxenstation.

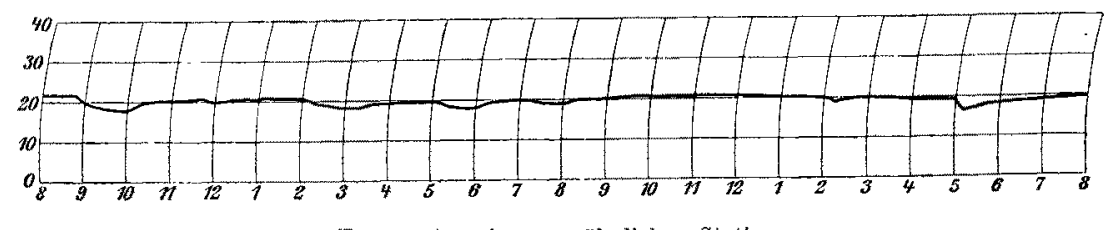

Temperatur einer gewöhnlichen Station.

Fig. 1.

sprach der $\mathrm{CO}_{2}$-Gehalt daher den Anforderungen $0,6-9^{\%} / 00$; in den übrigen, besonders in den fern von dem Abzug gelegenen Boxen stieg er aber bisweilen auf unzulässige Höhe - bis auf $1,26 \%$. Eine Verlegung des Ventilationsschachtes ist hier geplant.

Die Temperatur des Zimmers ist infolge der sorgfältigen Dosierung der Zu- hnd Abströmung äußerst gleichmäßig. Lang fortgesetzte Dauermessungen ergaben kaum eine Bewegung in der Temperaturkurve. Die obige sich über 24 Stunden erstreckende Temperaturkurve zeigt die Monothermie der Station (Fig. 1). Im ganzen liegt die Temperatur des Zimmers etwas höher als auf anderen Stationen. Da die Temperaturregulierung durch die Wärterin stets schwierig zu handhaben ist, soll in Zukunft die Thermoregulation der Warmwasserheizung automatisch bewerkstelligt werden. Besonders in der Zeit der Sommerhitze stieg die Temperatur höher als auf anderen Stationen. Durch Öfnen einer 
mit einem Gazerahmen überspannten Gitterklappe (vgl. S. 245 u. 246) im Fenster konnte dann Abhilfe geschafft werden.

Die relative Feuchtigkeit im Zimmer, gemessen am Haarhygrometer, unterschied sich nicht wesentlich von der in den übrigen Zimmern herrschenden.

Zur Entfernung der sich in Säuglingsstationen stets anhäufenden und bei der verlangsamten Ventilation besonders bemerkbaren üblen Gerüche wurde der Fußboden nach dem Czernyschen Vorschlag jeden Morgen einmal mit 1 proz. Lösung von Antiformin aufgewaschen.

Die Isolierung der Kinder geschah in 10 nach dem Mittelgang hin offenen Boxen, deren Wände wenige $\mathrm{cm}$ über dem Fußboden frei ließen und eine Höhe von 2,50 m erreichten. Die erste Boxenwand nach dem Fenster und die letzte nach der Tür wurde noch etwas höher angelegt. Die Boxenwände bestanden zunächst aus einer, in einen Holzrahmen gespannten Lage feinen Mulls. Eine Passage von Keimen durch die Gaze war nicht zu fürchten, denn nach den Untersuchungen Kobra ks ${ }^{1}$ ) hält feiner Mull alle Keime zurïck, die in einer Entfernung von $50 \mathrm{~cm}$ von dem Mull durch Hustenstöße ausgeschleudert werden.

Im Gebrauche stellte sich es bald heraus, daß die vielen Mullwände das Zimmer, namentlich in seinem hinteren, vom Fenster entfernten Teilen allzusehr verdunkelten. Es wurde daher in jede einzelne Boxenwand ein Glasfenster von $0,80 \mathrm{~m}$ Höhe und $1,15 \mathrm{~m}$ Breite eingesetzt.

Anfangs befürchteten wir, daß die Mullwände Fänger und Bewahrer von Krankheitskeimen wären, wenn wir nicht durch desinfizierende Lösungen täglich die der Gaze aufsitzenden Keime vernichteten. Wir bespritzten deshalb sämtliche Mullwände täglich einmal mit einer dünnen Sublimatlösung $1: 5000$. In der Tat wurde so. die Abtötung lebender Keime erreicht (z. B. fanden sich in $10 \mathrm{qcm}$ Mull vor der Sublimatbenetzung $\mathbf{7 6 0}$ Keime, nach derselben 0). Aber es kam dadurch zu einer unerwünschten Feuchtigkeitsüberladung der Luft, die um so mehr vermieden werden mußte, als dabei vielleicht nicht unschädliche Quecksilberdämpfe verbreitet wurden. Erfreulicherweise zeigte sich, daß die Befürchtung einer Keimbewahrung durch die Mullwände in der Tat nicht zutraf. Wie durch Keimzählung im städt. Untersuchungsamt festgestellt wurde, fand die Keimvermehrung nur in den ersten Tagen nach der Aufspannung des Mulls statt. Dann blieb die Zahl der nachzuweisenden Keime tagelang ungefähr auf gleicher Höhe, wahrscheinlich deshalb, weil stets ein Teil der Keime zu Boden fiel und so unschädlich gemacht wurde.

1) Respiratoren zum Schutze gegen die Einatmung infektiöser Tröpfchen and Stäubchen. Zeitschr. f. Hygiene u. Infektionskrankh. Bd. 68, S. 157. 




Fig. 2. Die Wubluxenstation

Zahl der Keime in 10 qon Mull

\begin{tabular}{|c|c|c|c|c|}
\hline nach & $\begin{array}{l}\text { Hohe } \\
2 \text { Tayen }\end{array}$ & $\begin{array}{c}18 \mathrm{~cm} \\
20\end{array}$ & $\begin{array}{c}1,414 m \\
167\end{array}$ & $\begin{array}{l}2.13 \mathrm{n} \\
280\end{array}$ \\
\hline : & 1 Tagen & 20 & 220 & 360 \\
\hline ox & 6) Tagen & 960 & 1720 & $10 \geq 0$ \\
\hline :" & + moehen & 230 & 1700 & - \\
\hline
\end{tabular}


Wir sahen daher ron der Sublimatckinfektion der Mullwinde als, wnd begü̈gten uns mit einen alle 4 Wochen stattindendea Fechsel dus Mulls.

Nicht unjuteregsant ist die Tatsache, dab sich dio grobte Feimzahl stets in jener Mullage fand, die dem Kopfo dar Kinder am nächsten war (in der Hohe won $1,06 \mathrm{~m}$ ). Man darf diese Keimansammlung wohl auf die mit den Husteaistößen stattfindende Keinausstreuung zurückführen und dies mit um so gröberex Berechtigung, als bei stark hustenden Kindern in eben derselben Höhe stets viel mehn Weime gefunden wurden, sh bei gesunden.

$\begin{array}{ccc}\text { Keimaln in } 10 \text { qcm Mull in } 1,06 \text { Tohe } \\ \text { bei Gesunden } & \text { bei Hustentes } \\ \text { 1. Tag } & 987 & 1040 \\ \text { 2. Tag } & 840 & 6080 \\ \text { 3. Tag } & 3920 & 6560\end{array}$

Die Mullwände wurden bei dex später eingerichteten Station (Station 7) durch Holz-Glaswände ersetzt (Fig. 3). Zur Zeit, in der ich diesen Bericht niederschreibe, bin ich allerdings nicht imstande, diese Anderung auch als Verbesserung zu bezeichnen. Mag auch der Schönheitssinn firr die Claswände entscheiden, so sprechen doch, wenigstens bei den vorliegenden System der Za- und Ablüftung gewichtige Cründe für die Beibehaltung der zunächst nur aus ökonomisehen Gründen gewählten Mullwände. Die durch clie Fensteröffnung eingetretene Luft passiert hier langsam, mehr und mehr in der Stromstärke gehemmt, Boxe auf Boxe, bis sie durch die Ventilationsklappe auf der dem Fenster entgegengesetzten Wand des Zimmers entweicht. So erhält jodes Kind seinen Anteil an der frischen Luft, ohne daß eine Verschleppung von Krankheitskeimen duroh zu starke Luftströmungen zu befürchton steht. Anders bei den Glaswänden. Schon dic erste, dicht beim Fenster gelegene Glaswand verhindert den direkten Durchtritt der frischen Luft in die erste Boxe. so $\mathrm{da} B$ der Hauptluftstrom gezwungen wird, entweler den Mittelgang oder den Weg unter oder oberhalb der Boxe zu gehen. Die Innenluft innerhalb der Boxe selbst Kam so unter Umständen einige Zeit stagnieren oder an der Ventilation nux unregelmäßig Anteil nehmen. Un die GleichnäBigkeit der Luftemenerung in den Glasboxen zu erzielen. wird wahrscheinlich ein komplizierteres Ventilationssystem erforderlich sein, in der Weise, daß jede Boxe für sich eine Ventilationsvorrichtung für Zuführung oder zumindest für Abführung der Luft erhält. Eine solche Anlage ist für eine demnächst zu errichtende Boxenstation geplant.

Das Gesamtbild der Stationen, alle Finzelheiten der Kinrichtung (Wusse der Boxenwände usw.) erkennt man aus den beigegebenen Bildern and Zeichnongen in Quer- und Frontalschnitt. (Figuren 2, 3, 4. u. 5.) 


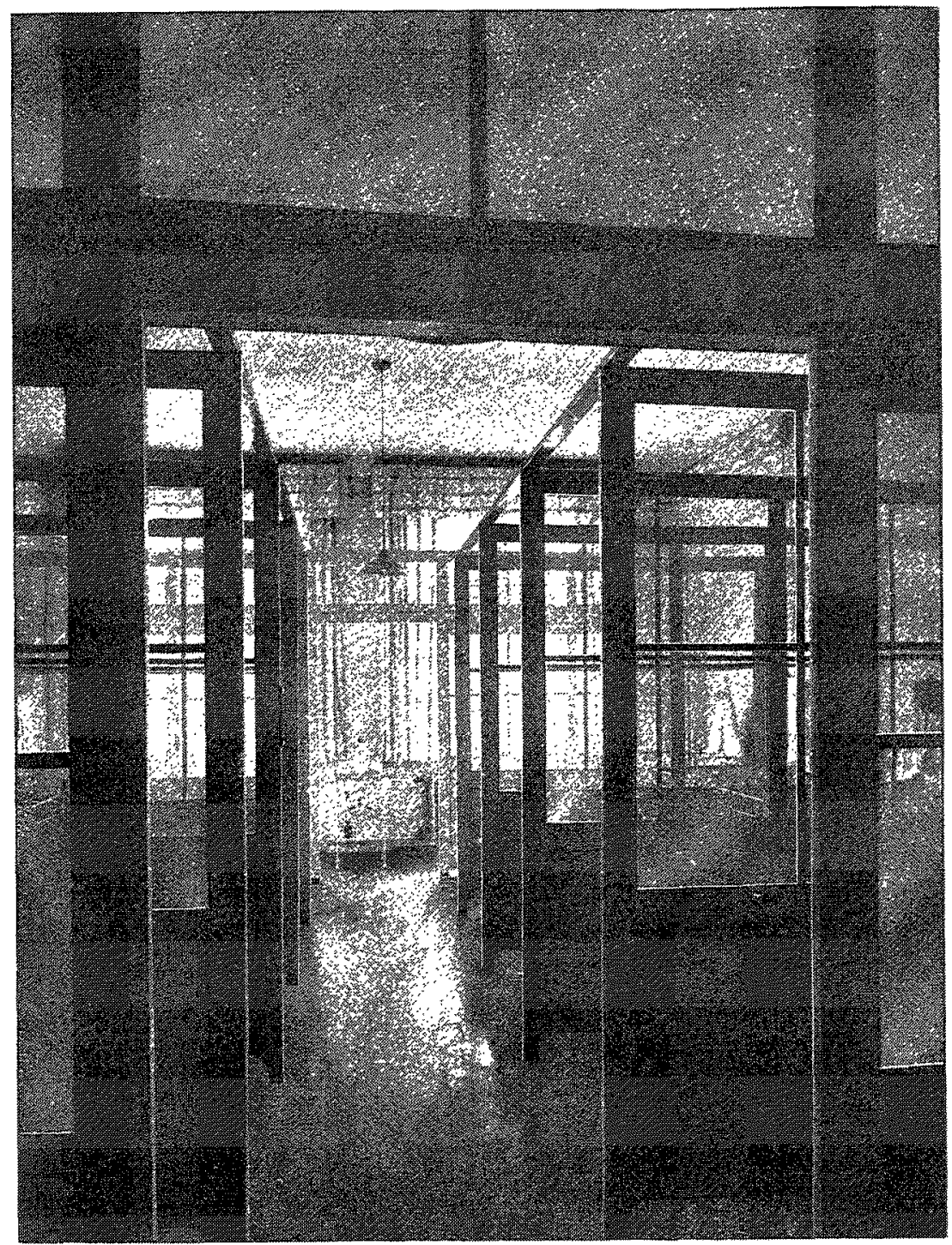

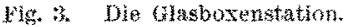

AuBex den das ganze Kind isolierenden Vollboxen wurde noch eine eintachere Art der Isolierung ebenfalls in Kombination mit den erwähnten Ventilationscinrichtungen versucht. eine Isolierung des für die Obertragung gefahrlichsten Teiles des Kindes, des Kupfes, durch sogenamnte Kopfboxen. Auf einer Station (ro) wurden alle Insassen ständig unter diesen Kopfboxen gehalten. 

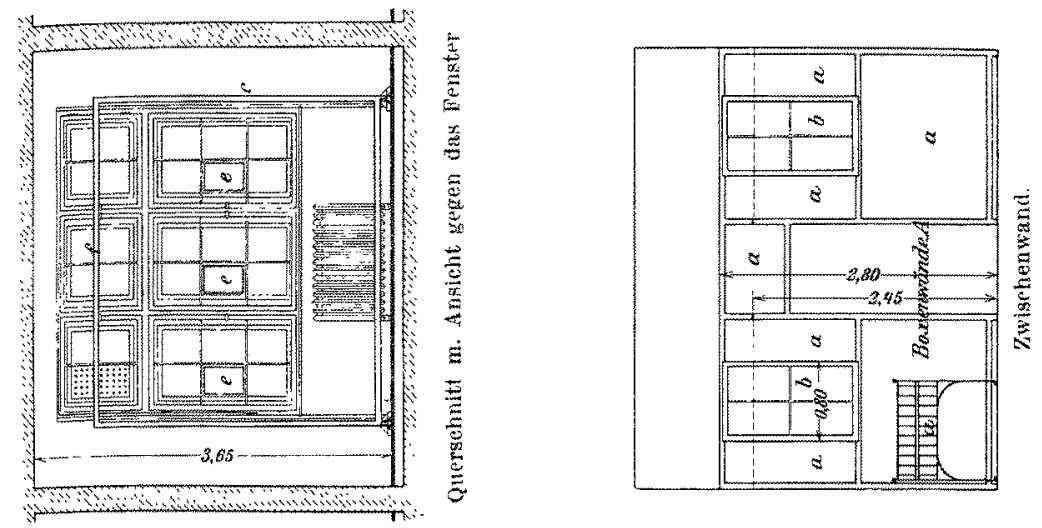

茯节
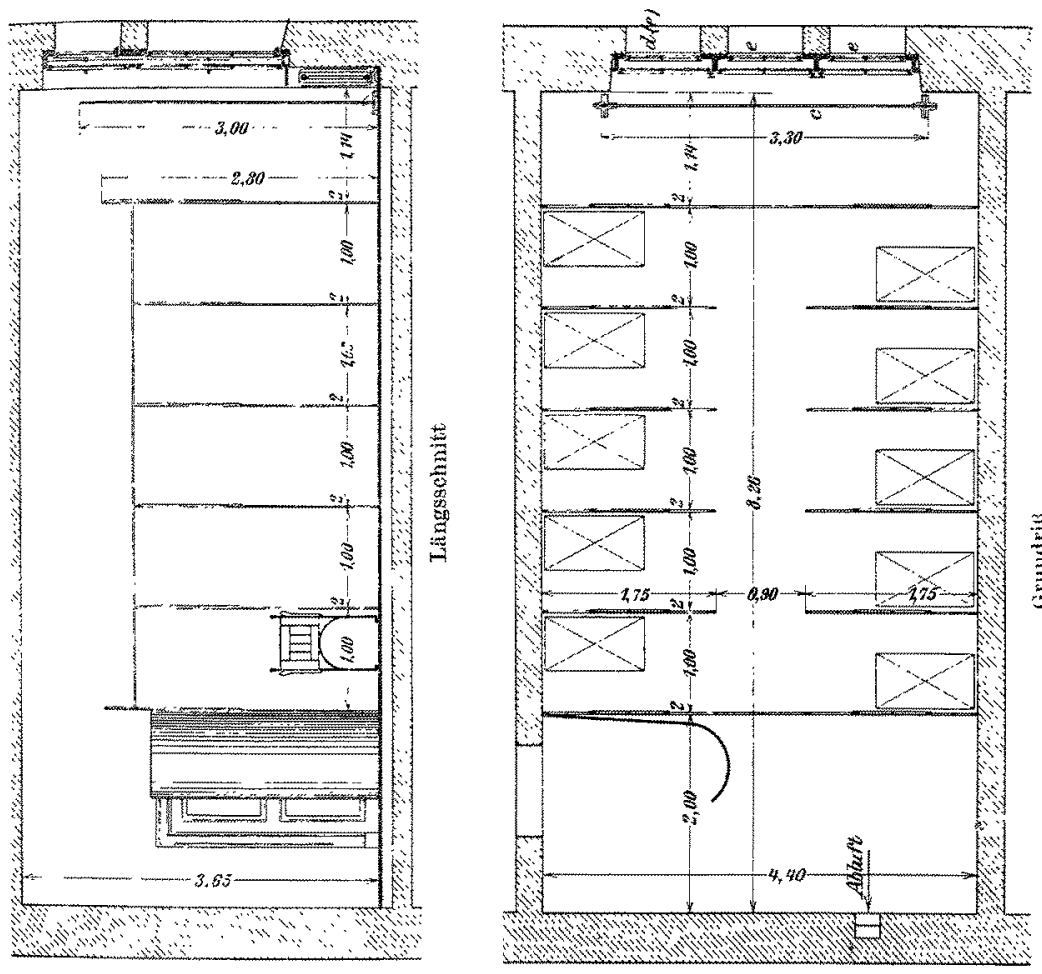

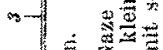

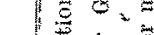

; 1 整高

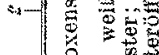

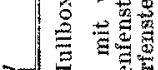

-

$+\mathrm{E}$

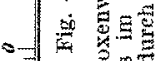

-

3 a $=$

e

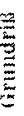

蛋

落:

.

范

的政

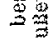

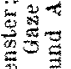

政

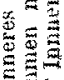



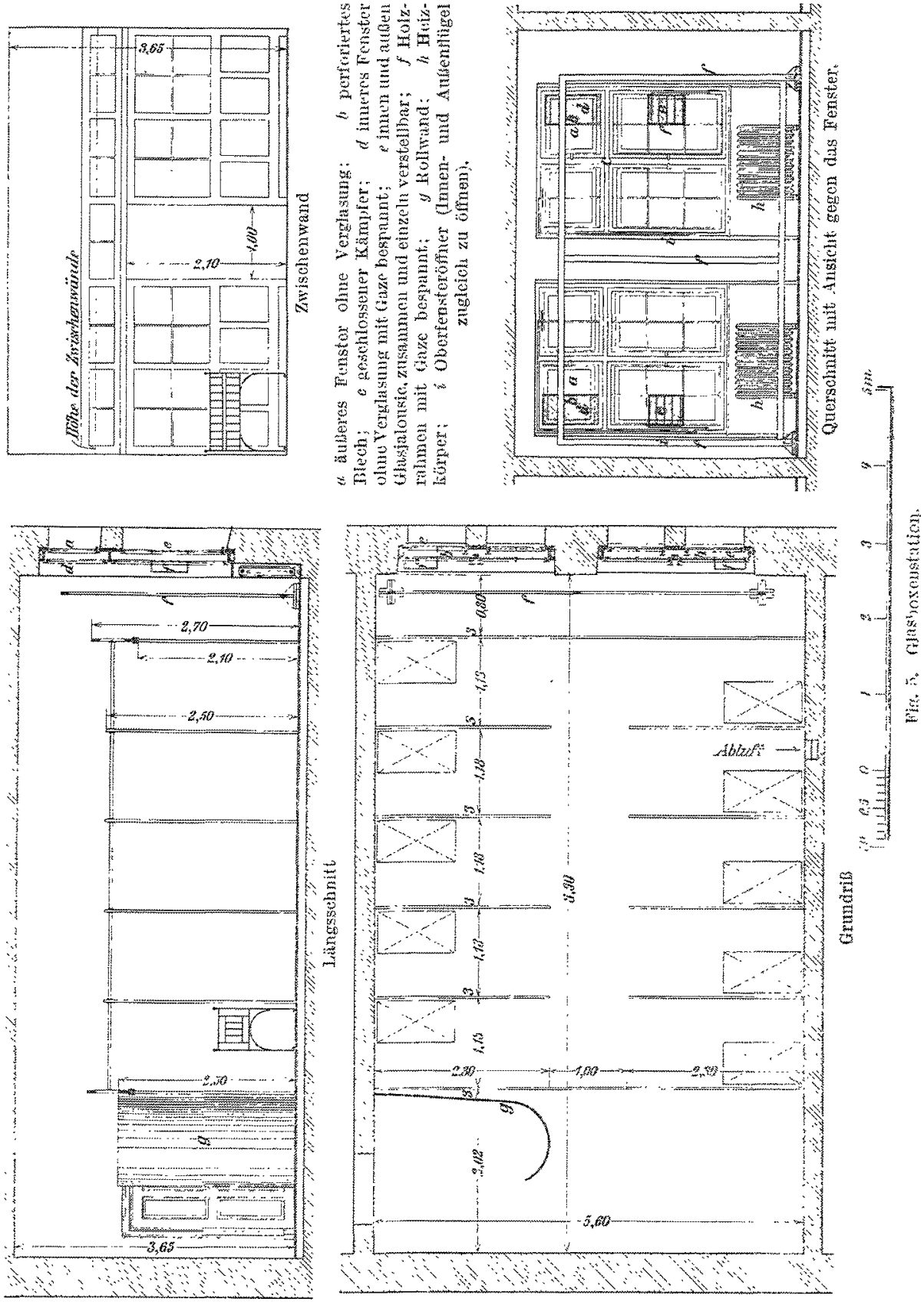
Wie die lolgende Skizze ohne weiteres erkennen läbt (Fig. 6), bestent die Kophboxe aus enem leicht zusammensteckbaren Holzrahmen, der an den Seiten mit feiner alle 4 Wochen austuwechsender Gaze uberkleidet ist wnd nach oben mit ener drrchsichtigen ganz dünon Celluloidplatte überdeckt ist. In die Celluloidplatte ist an einer Ecke wur Erzielung einer besseren Durchlütung ein kleines dreieckiges Fenster ausgeschnitten. Die Kopfboxe wird am Koptende des Bettes so autgestellt, daß der Kopf des Kindes durch sie völig von der Umgebung abgeschlossen ist. Bei dem Ferausnehmen des Kindes ist es nur notwendig, den an der Vorderseite der Koptboxe befindichen Mullvorhang aufqunehmen. Die Nachteile, die einer solchen primitiven Vorrichtung innewohnen, bedüten kaum einer besonderen Erörterung. Sie bestehen in einer Ersehwerung der Beobachtung des. Kindes in einer vielleicht nicht ausreichenden Tuftzufuhr und infolgedessen dem Nachteil einer Kohlensäureanhafung und einer besonders zur heiBen Zeit eventuell entretenden Obererwärmung des Kindes. Diesen Nachteilen, die sich bei sorgfältiger Uherwachung zum Teil vermeiden lassen, steht der cine Vorteil der Beschränkung des rröpfchentransports gegenüber. Dab die Kopfboxe, solange sie allseitig geschlossen ist, Krankheitserregen keinen Durchtritt bietet, geht aus der. Tatsache hervor, dab anf dem Mull gelegentich Diphtheriebacillen aachgeviesen wurden. Ein ständiger Vorschlub der Kopfboxe, wie er zur Vexmeidung ron Infelionsibbertragungen Foranssetzung wäre, last sich aber schon ceshalb nicht durchsetzen, well bei jeder Futterung der Kinder, bei jeder Untersuchung, bei jedex Trockenlegung der Mullworhang zurückgeschlagen werden mue. Immerhin sind die Kopfboxen, mögen sie auch nur einen Notbehelf dar. stellen, hei dex Isolierwng stark husten-
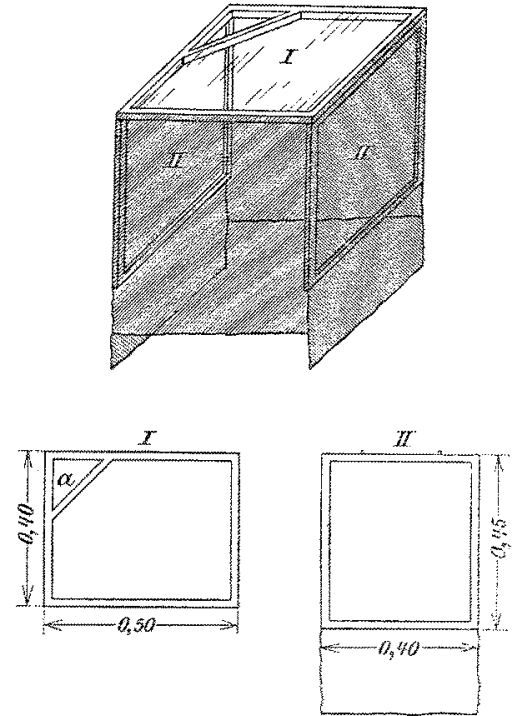

Fig. 6. Fopboxe.

I Oberew Rabmen mit 1 man starker durch. slohtiger Zeluloldplatte bokleidet mit Ausnahrae des Ureiecks a.

If Seiterteile, mit dem Ramen I duch je 2 Dikel ausinandemehrbar verbunden die 4 seitentächen sind mit feiner weiser

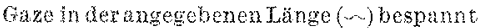

der, besonder auch tuberkulöser Kinder, somie nur Isolierung von Diphtheriebacllenträgen selbst in Stationen ohne besondere Ventilationseiarichtung nicht ohne Xuzzen.

Der Dienst axf den Stationen ging in der gewohnten Waise vor sich. Fede Plegerin hat ber Tage 10 , bei Nacht 20 Säuglinge zu versongen. Art sowohl als Plegerin waschen sich nach jeder Beruhrung eines Kindes die Wande mit Seife, ein Wechsel der Wäntel findet im allgemeinen nicht stat, nur bei der Versorgung von masem- und kevchhusten- 
kranken Kindern tragen Arzt und Pflegerin in der entsprechenden Boxe einen besonderen Mantel.

Da der Besuch unserer Pfleglinge durch Verwandte nicht verboten werden kann, dürfen die besuchenden Personen nur den Mittelgang der Boxenstation betreten und von dort aus, durch eine Kette von der Boxe abgesperrt, das Kind betrachten.

Im Falle grippaler Erkrankung des Arztes oder der Pflegerin wurde, wenn die Ausschaltung aus dem Dienste nicht zu ermöglichen war, das Tragen einer Mullmaske - leider gibt es noch nichts besseres vorgeschrieben. Stets wurde besonders darauf geachtet, daß innerhalb der Boxenstation durch langsame Bewegungen und ruhigen Schritt möglichst wenig Luftbewegung verursacht wurde.

Die Einrichtung der Boxenstation wurde nach ihrem heutigen Stand wiedergegeben. Es soll aber nicht verschwiegen werden, daß die Einrichtungen bei Eröffnung der Station Anfang November 1911 zunächst noch unvollkommener waren und erst allmählich mit wachsender Erfahrung ihre Ergänzung fanden. So erwies sich die Luftzufuhr durch das perforierte Fenster, die Aufstellung des Wandschirmes und der Mullgardine hinter dem Fenster erst allmählich als notwendig. Die Mullboxenstation (11) repräsentierte sich in der beschriebenen Form erst am 26. I. 12. Die Glasboxenstation (7) erhielt erst nach Abschluß dieses Berichtes aus einer noch später zu besprechenden Veranlassung heraus dieselbe Mullgardine hinter dem Fenster, wie Station 11. Die Kopfboxenstation (10) wurde am 24. I. 12 in Betrieb genommen. Der Bericht über alle 3 Stationen wurde am 1. XI. 12 abgeschlossen.

\section{Die Infektionsverhütung.}

A. Die Erkrankungen der Luftwege.

In erster Linie galt die Einrichtung der Boxenstationen der Verhinderung der Grippeübertragung. Wie ist sie dieser Aufgabe gerecht geworden?

Die Mullboxenstation passierten 75 Kinder mit einer Aufenthaltsdauer von durchschnittlich 35,8 Tagen, 36 Kinder wurden mit Erkrankungen der Respirationsorgane aufgenommen. Diese hohe Zahl katarrhalischer Erkrankungen erklärt sich daraus, daß mit Vorbedacht eine große Anzahl grippekranker Kinder auf die Boxenstation dirigiert wurden. 36 Neuaufnahmen mit grippaler Erkrankung haben sonst nach der vorher angeführten Morbiditätsstatistik 72 weitere derartige Erkrankungen der Anstaltsinsassen im Gefolge. Tatsächlich aber er- 
krankten in der ganzen Zeit nur 2 Kinder an Katarrhen der Luftwege, als deren Ursache naturgemäß auch eine Infektion von seiten der Besucher, des Arztes oder der Pflegerin nicht auszuschließen ist. Wenn man aber selbst eine. Infektion durch andere Stationsinsassen zugibt, so muß doch angesichts dieser überaus geringen Zahl von Neuerkrankungen der Erfolg der Einrichtung als ein guter bezeichnet werden.

Die Glasboxenstation passierten 31 Kinder mit einer durchschnittlichen Aufenthaltsdauer von 25 Tagen. 16 davon waren an Grippe erkrankt. Bis zum Abschluß dieses Berichtes wurde keine Utbertragung beobachtet. Erst im Laufe des Novembers erkrankten 4 Kinder gleichzeitig mit Katarrhen, die augenscheinlich auf eine Infektion zurückzuführen waren. Die Erkrankungen gaben den Anlaß zu der vorher bereits erwähnten Korrektur der Luftzuströmung durch Aufstellung der Mullgardine, die wir zunächst entbehren zu dürfen glaubten. Nach diesem Zeitpunkt haben wir bis heute keine weiteren Erkrankungen während des Aufenthaltes der Kinder auf der Station erlebt.

Die Kopfboxenstation war bis zu dem AbschluB des Berichtes mit 65 Kindern belegt, deren durchschnittlicher Aufenthalt 43 Tage währte. 25 davon waren mit Katarrhen der Luftwege behaftet. Auf dieser Station wurden 10 Neuerkrankungen beobachtet. Und zwar im Februar 3, im April 4, im Mai 1, Juni 1, August 1. Die Erkrankungen im Februar und April waren vielleicht Folgen einer Übertragung durch die an heftiger Grippe leidenden Pflegerinnen, die nicht vom Dienste befreit werden konnten. Immerhin blieben selbst nach Abrech. nung dieser Utbertragungen durch die Pflegerin noch 3 Neuerkrankungen während des Aufenthaltes auf der Station übrig. Die Einrichtung der Kopfboxen hat dem. nach einen schwächeren Schutz gegen die Übertragung der Grippe gewährt, als die der Vollboxen.

Mit besonderem Interesse mußte man den Verlauf der grippalen Erkrankungen innerhalb einer Station, in der Neuinfektionen fast völlig vermieden waren, verfolgen. Das Interesse galt vor allem der Beantwortung der Frage, ob die häufigen Rezidive der Katarrhe der Luftwege, wie man sie sonst auf Säuglingsstationen zu sehen gewohnt ist, nun ausbleiben oder ob sie selbst in diesem so gut wie infektionsfreien Milieu wieder aufflackern, ob mit anderen Worten die rezidivierenden Erkrankungen die Folgen exogener oder endogener Reinfektion sind. Die Beantwortung der Frage wurde noch dadurch erleichtert, daß Kinder mit immer wieder rezidivierenden Katarrhen von anderen Stationen auf die Boxenstationen verlegt wurden.

Nach dem Verlaufe der grippalen Erkrankungen auf der Boxenstation ist eine Einteilung in zwei verschiedene Krankheitsformen erlaubt, 
in eine im infektionsfreien Milieu zu endogener Reinfektion ncigende Form und in eine nicht zu endogener Reinfektion neigende Form.

Die erste Erkrankungsart, die sich endogen reinfizierende, ist selten. Im ganzen haben wir nur 4 derartige Fälle beobachtet. Dreimal handelte es sich um Kinder mit Pneumonie (einmal neben schweren hämorrhagischen Masern), von denen eins (das Masernkind) in der zweiten Woche des Aufenthalts in der Station an Pneumonia migrans starb. Die beiden

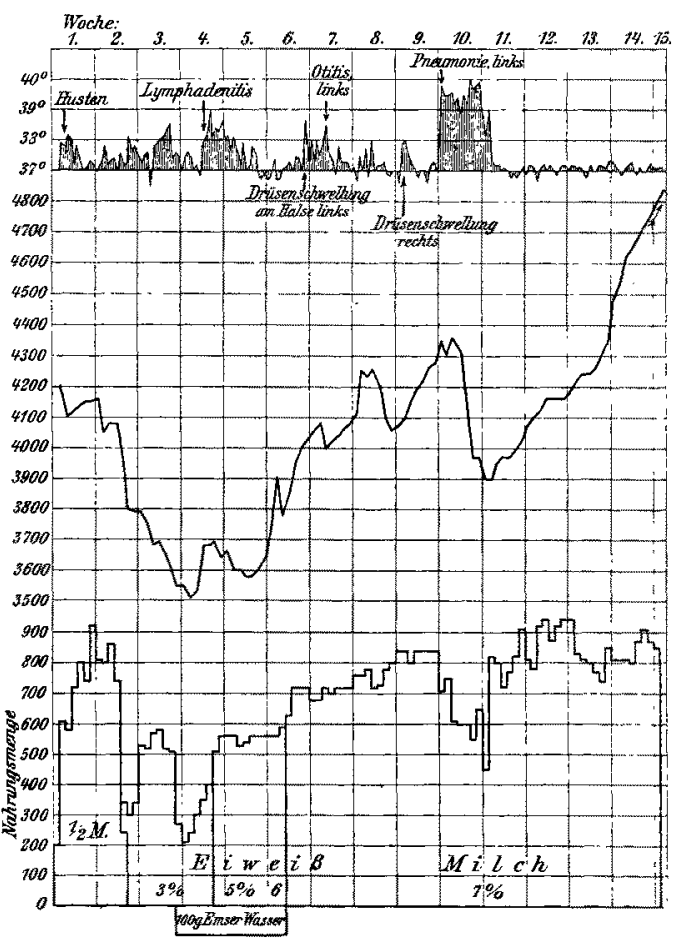

Fig. 7.

anderen erkrankten nach 2 resp. 7 tägigem fieberfreiem Intervall erneut an Pneumonie. Der 4. Fall betraf einen Säugling mit 6 Wochen dauerndem Schnupfen, der in dieser Zeit häufig zu mäßigen Fieberzacken führte. Als typisches Beispiel dieser rezidivierenden Form der Erkrankung sei folgender (nach dem 1. XI. beobachteter) Fall mitgeteilt.

E. R. (Fig. 7), 2 Monate alt, aufgenommen mit Husten, Pharyngitis und Fieber, in der 4. Beobachtungswoche Entwicklung einer Lymphadenitis. Zuerst stärker auf der linken Halsseite, gleichzeitig Otitis links, in der 9. Woche stärkere Drüsenschwellung rechts. In dor 10. Woche Pneumonie. In der ganzen Zeit also 10 Wochen lang - entweder beträchtliches Fieber oder subfebrile Tempe- 
raturzacken. Erst nach dem Abklingen der Pneumonie endgültiger Abfall der Temperatur zur Norm und Gedeihen.

Erfahrungen dieser Art machen es wahrscheinlich, daB die Krankheitserreger sich wochenlang in Körper aufhalten und ohne Hinzutritt weiterer exogener Infektionen immer wieder zu neuen Manifestationen führen können, bis es der wachsenden Immunität des Organismus gelingt, die Krankheitserregerunschädlich zu machen.

Das Gros der Fälle gehörte indes der zweiten Form der Erkrankung an: Rascher Ablauf der katarrhalischen Erkrankung innerhalb weniger Tage und später bei Vermeidung neuer Infektionen Freiheit ron Rezidiven. Interessanterweise finden sich gerade unter diesen Kindern einige, die wochen- und monatelang im infektionsschwangeren Milieu anderer Stationen unaufhörlich von katarrhalischen Erkrankungen heimgesucht wurden und bereits in den bedrohlichsten Zustand geraten waren, nun aber durch die Bewahrung vor neuen grippalen Infektionen ohne Än-

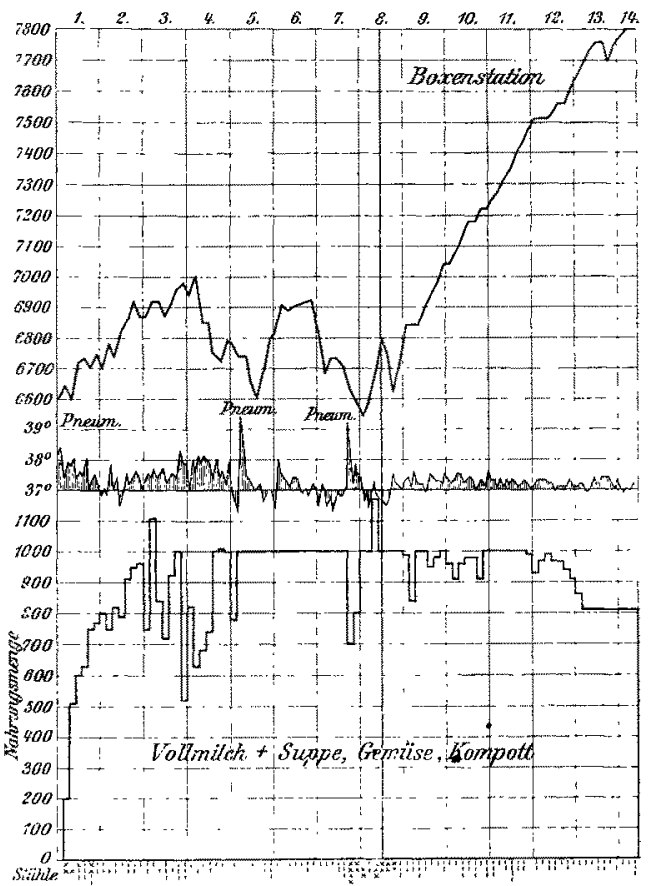

Fig. 8 .

Kind L. 1 Jahr. In $7^{1}$. Woehen 3 Erkrankungen an Preumonie auf einer gewöhnlichen Station des Hauses. Jedesmal schwere Alteration des Ernährungsaustandes, so daß jeder Fortschritt vereitelt wird. In der 8 . Beubachtungswoche verlegung auf die Boxenstation: Ungestörtes Gedeihen, das auch in der Außen. pflege anhält (vgl. Fig. 9).

derung der Ernährungsweise sofort zu gutem Geleihen gebracht wurden. Wiederum seien zwei Beispiele kurz mitgeteilt und mit. Kurven belegt. Fig. 8, 9, 10.

Ebenso wie in diesen beiden Fällen die Temperatur mit der Verlegung auf die Station nach langer Fieberbewegung zur Norm sank, so war dies in einer ganzen Reihe von Fällen bisweilen in geradezu lrritischer Weise zu beobachten. 




Nicht ganz leicht zu beantworten ist die Frage, ob das Freibleiben von weiteren Erkrankungen lediglich die Folge der Vermeidung exogener Infektion ist oder ob der Wegfall exogener Schädigungen anderer Natur dabei im Spiele ist. Um die Ubertragung infektiöser Keime zu vermeiden, mußten wir Einrichtungen treffen, die gleichzeitig vielleicht in ganz anderer Richtung liegende Schädlichkeiten hintan- 
halten: Kann, um nur ein Beispiel anzuführen, durch die Gleichmäßigkeit der Wärme in der Station sowie durch das Fehlen jedes schärferen Luftzuges nicht eine günstige Beeinflussung auf die Respirationsorgane ausgeübt werden, die diese einer Erkrankung weniger geneigt machen? Erlaubt demnach das zeitliche Zusammentreffen zwischen der Einrichtung infektionsverhütender Maßnahmen und der tatsächlich erreichten Vermeidung katarrhaliseher Erkrankungen auch nicht die unbedingte Folgerung auf einen Kausalkonnex zwischen beiden, so dürfte es doch nicht zu gewagt sein, wein man diesen kausalen Zusammenhang als wahrscheinlich bezeichnet.

\section{B. Pertussis.}

Keuchhustenkranke wurden ausschließlich in den beilen Vollboxenstationen aufgenommen. Der Keuchhusten gehört - in unserer Anstalt - zu den am meisten gefürchteten kontagiösen $\mathrm{Er}$ krankungen. Nicht selten haben wir, besonders als wir die Epidemiologie der Pertussis

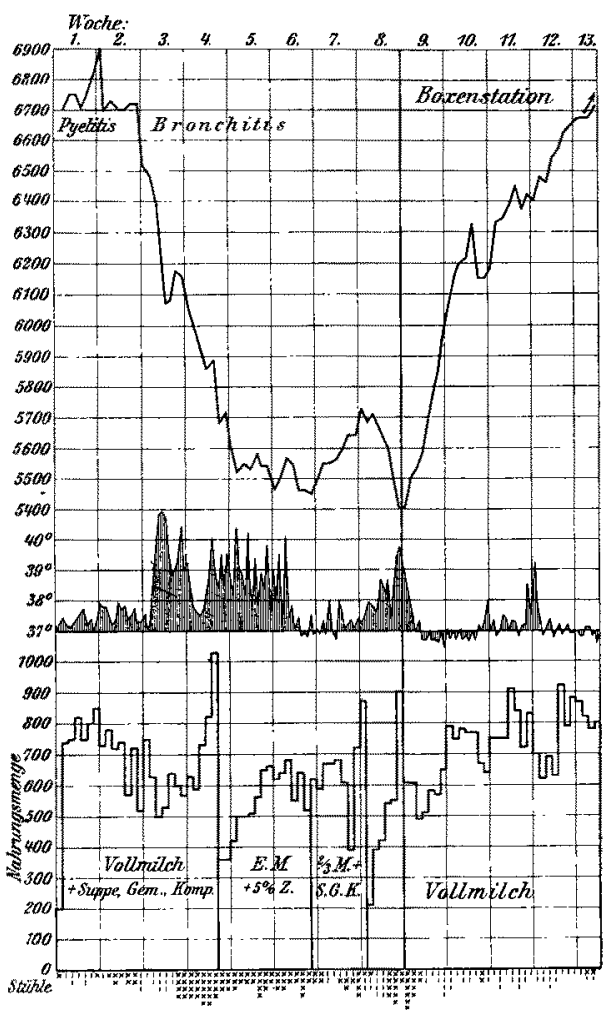

Fig. 10.

W. 10 Monate alt, sehwere Pyelitis, die sich durch Hinzutritt einer Bronchitis wärend des Aufenthaltes des Kindes auf einer gewöhnlichen Station sehr verschlechtert. Parenterale Stôrung mit stakken Gewichtsstirzen. - Verlegung in die Boxenstation zu Beginn der 9. Beobachtungswoche. Sofortige Erholung, Ablingen der Bronchitis und Gewichtszunahme. Gelegentlich noch durch die Pyelitis bedingte Fieberzacke. In der 13. Woche im Urin kein krankhafter Befund mehr. Entlassung. Fernerhin ungestörtes Gedeihen. noch nicht so wie heute beherrschten, völlige Durchseuchung einzelner Krankensäle erlebt. Nicht alle Anstaltsärzte haben bekanntlich ebensolche Erfahrungen über die Infektiosität des Keuchhustens machen müssen. Czern y ist sogar auf Grund seiner reichen Erfahrungen der Meinung, da $\beta$ eine Vermeidung der Keuchhustenübertragung ausschließlich durch eine größere Distanz der Betten $(1,50 \mathrm{~m}$, ebenso wie er sie zur Vermeidung der 
Grippeübertragung fordert) zu erreichen ist. Ohne - mangels einschlägiger Beobachtungen - auf diese Frage einzugehen, sei über die Resultate der Unterbringung Pertussiskranker in unseren Boxenstationen berichtet. Im ganzen passierten die Stationen 7 und 11 bis zum 1. XI. 20 keuchhustenkranke Kinder, eine Zahl, die sich bis heute noch beträchtlich weiterhin erhöht hat. Eine Ubertragung der Pertussis auf Stationsinsassen haben wir nicht erlebt. Als besonders bemerkenswert möchte ich die Milde des Verlaufs der Keuchhustenerkrankung hervorheben. Sowohl die Stärke als auch die Zahl der Anfälle nahm in einer Reihe von Fällen so rasch $a b$, daß man nicht umhin kann, dem ,Klima" der Boxenstation vielleicht bedingt durch die gleichmäßige Wärme und durch das Fehlen aller auf die Respirationsschleimhaut wirkenden äußeren Reize - einen günstigen Einfluß aus den Verlauf des Keuchhustens zuzuschreiben. Sicherlich ist allerdings auch das Freibleiben von komplizierenden Infektionen für den Ablauf des Keuchhustens von großer Bedeutung. Nach 2-3wöchentlichem Aufenthalt in der Boxenstation waren die Anfälle oft so gering geworden, daß die Kinder in Außenpflege entlassen werden konnten. Als Beispiel dieser Art sei das Kind W. W. angeführt. Geboren 10. II. 12, angenommen 16. X. 12. Bei der Aufnahme frischer mittelschwerer Keuchhusten.

Anzahl der täglichen Anfälle:

\begin{tabular}{|c|c|}
\hline 18. X. . . 22 & 24. X. \\
\hline 19. X. . . . 15 & 25. $X$. \\
\hline 20. X. . . . 10 & 26. X. \\
\hline X. . & 27. $\mathrm{X}$. \\
\hline $\mathrm{X}$. & 28. $X$. \\
\hline
\end{tabular}

C. Masern.

Nach den günstigen Erfahrungen bei Grippe und Pertussis durfte man auch den Versuch mit der Aufnahme Masemkranker machen. Die Masern sind bekanntlich in Säuglingsstationen wenig zu fürchten, weil Kinder unter 4 Monaten ihnen gegenüber eine hohe Immunität besitzen. Um zu beweisen, daß die Boxen tatsächlich einen wirksamen Schutz gegen die Masemübertragung gewähren, muß·daher die Anwesenheit krankheitsbereiter Individuen vorausgesetzt werden. Tatsächlich wurden zur Zeit, als Masernkranke auf der Station waren, mehrere ältere Säuglinge auf der Station verpflegt, z. B. im März 1 Kind mit 1 Jahr, 2 Kinder mit 6 Monaten und 1 Kind mit 5 Monaten; und im 
September, als gleichzeitig 2 Masernkranke auf der Station lagen, 3 Kinder mit 5, 3 Kinder mit 6, 9 Monaten und 1 Jahr.

5 mal wurden Masernkranke aufgenommen, eine Krankheitsübertragung fand nicht statt. Die einfachen Maßnahmen bezüglich der Verhütung von Luftübertragung scheinen also eine Weiterverbreitung der Masern verhindert zu haben. Wenn ich diesen Satz noch mit einer gewissen Vorsicht ausspreche, so geschieht es einerseits, weil die Zahl unserer Erfahrungen noch gering ist (wenn sie auch inzwischen noch vermehrt, wurden), andererseits, weil die Masernerkrankung unserer $\mathrm{Pa}$ tienten bei der Aufnahme stets schon auf der Höhe angelangt war, in der sie weniger infektiös ist, als in der Inkubationszeit der Erkrankung ${ }^{1}$ ).

\section{Diphtherie.}

Die Ausbreitung der Diphtherie hat in Berlin, wie bereits mehrfach berichtet wurde, in clen letzten zwei Jahren erheblich zugenommen; im besonderen Maße haben darunter die Insassen der Säuglingsspitäler zu leiden. Denn wie jüngst Schloß und Selig $m a n n^{2}$ ) nachgewiesen haben, kommt eine große Anzahl von Säuglingen als Bacillenträger (nach Seligmanns letzten Untersuchungen $12 \%$ aller Aufnahmen) ins Haus und überträgt die Diphtheriebacillen auf vordem bacillenfreie Kinder. Jeder Bacillenträger ist aber der Gefahr einer Erkrankung an Diphtherie ausgesetzt, sei es, daß sich die Diphtherie primär entwickelt oder sekundär auf einen bestehenden Katarrh der Luftwege aufpfropft. Und wenn auch die schweren Erkrankungen an Rachen- oder Kehlkopfdiphtherie glücklicherweise selten zu beobachten sind (im letzten Jahre zählten wir 11 Rachendiphtherien neben 65 Nasendiphtherien), so ist doch auch der diphtherische, meist blutig-seröse, Schnupfen nicht leicht zu nehmen. Eine Bekämpfung der Diphtherieübertragung wird nur dann erfolgreich sein, wenn es gelingt, die Bacillenausscheider wirksam zu isolieren. Leider hat unser Boxensystem die Úbertragung von Diphtheriebacillen auf gesunde Kinder nicht verhindern können.

Die Untersuchung auf Bacillenträger wurde nicht immer regelmäßig durchgeführt. Im Laufe des Monats September wurden die Insassen der Mullboxenstation regelmäßig jede Woche auf Diphtheriebacillen untersucht. Von 6 in die Station aufgenommenen Bacillenträgern

1) Anm. b. d. letzten Korrektur: Wie notwendig diese Reserve war, geht daraus hervor, daß in den letzten Tagen des März von einem im Prodromalsta di u m der Masem (noch vor dem Erscheinen der Koplikschen Flecke) auf die Boxenstation aufgenommenen Kinde aus eine Übertragung der Erkrankung erfolgt ist.

2) Zeitschr. f. Kinderheilk. 4. 1912. 
wurde 1 gesundes Kind mit Bacillen infiziert. Auf der Glasboxenstation wurden in einem Zeitraum von 8 Wochen allwöchentlich in Nase und Rachen der Kinder auf Diphtheriebacillen gefahndet. 5 mit Diphtheriebacillen eingelieferte Kinder übertrugen ihre Bacillen in diesem Zeitraum auf 15 weitere Kinder. 3 mal kam es dabei zum Ausbruch des als diphtherisch anzusprechenden blutig-serösen Schnupfens.

Zur selben Zeit wurden auch die Kinder auf der Kopfboxenstation einer wöchentlich wiederholten Untersuchung auf Diphtheriebacillen unterzogen. Innerhalb 6 Wochen wurde von 4 aufgenommenen Bacillenausscheidern hier 4 weitere Kinder infiziert.

Diese Utbertragungen zeigen also, daß bei der Verschleppung der Diphtheriebacillen der Transport durch die Luft eine geringe Rolle spielt. Wahrscheinlich muß die Pflegerin als Vermittlerin der Übertragung angesehen werden. Tatsächlich erkrankte während der Untersuchungszeit die Pflegerin der Kopfboxenstation an Rachendiphtherie, während die Pflegerin der Glasboxenstation als Diphtheriebacillenträgerin ermittelt wurde. In jedem Falle kann von der Einrichtung der Boxenstation nach unseren bisherigen Erfahrungen ein Schutz gegen die Verbreitung der Diphtheriebacillen nicht erwartet werden.

\section{E. Varizellen.}

Die Erreger der Varizellen verbreiten sich nach allgemeiner Erfahrung außerordentlich leicht. Schon der kürzeste Aufenthalt eines an Windpocken erkrankten Kindes in der Station genügt, um die Erkrankung aller bisher von Windpocken verschonten Kinder zu bewirken. An der hohen Ubertragbarkeit des Windpockenvirus ist selbst das sonst. so gut funktionierende System Les a ges gescheitert, denn nach Lesages Bericht wurden Varizelleninfektionen nicht vermieden. Ein Zufall fügte es, daß wir die Leistungsfähigkeit der Glasboxenstation gegenüber der Verhinderung der Varizellenübertragung erproben muBten. Es erkrankte nämlich am 25. IX. ein kurz vorher aufgenommenes Kind an Varizellen. Rasch genug waren wir in der Lage, das Versagen unserer Boxenstation festzustellen; denn schon am 16. X. erkrankte ein weiterer Pflegling der Station an Windpocken und fernerhin bis zum 26. XI. im ganzen 5. Nicht uninteressant ist die Verfolgung des Weges der Infektion. Die erste Erkrankung betraf ein dem Varizellenfall gegenüberliegendes Kind, die 2., 3. und 4. ebenfalls Kinder der gegenüberliegenden Seite, die 5. und letzte erst den Nachbar. Diese Reihenfolge der Erkrankungen spricht gegen die Kontaktübertragung und für die Übertragung auf dem Luftwege im Sinne von Lesage, weil 
sich die Infektion in 4 von 5 Fällen in der Richtung des Luftstromes nach der Abzugsklappe an der gegenüberliegenden Wand verbreitete (vgl. nebenstehende Skizze).

Auch in anderen Stationen haben wir seitdem gelegentlich von Varizellenübertragungen schonzweimal eine Reihenfolge der Erkrankungen feststellen könmen, wie sie der Keimver-

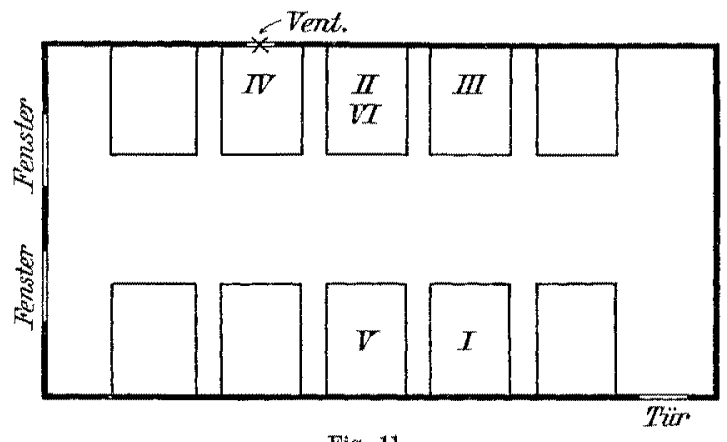

Fig. 11. schleppung mit der Luftströmung vom Fenster zur Türe entspricht.

\section{Ernährungsresultate auf den Boxenstationen.}

Ist die Erkrankung der Luftwege mit ihrer Einwirkung auf den Ernährungszustand tatsächlich die Hauptursache des Hospitalisnuus, wie er sich heute noch geltend macht - eine Auffassung, die von Finkelstein in seinem Lehrbuche vertreten wird - dann mub der Wegfall dieser Erkrankungen die Ernährungsresultate in bemerkenswertester Weise beeinflussen: Insbesondere müssen die Durchfälle künstlich ernährter Kinder seltener werden und die Durchführung der künstlichen Ernährung neugeborener und junger Säuglinge mit geringeren Schwierigkeiten als bisher verknüpft sein. Sind diese Erwartungen eingetroffen?

In der Tat war bei Ernährung mit gewöhnlichen Milchmischungen die Entleerung fester Stühle die Regel. Kamen Kinder mit häufigen Stïhlen auf die Boxenstation, so heilte nicht selten der Durchfall binnen wenigen Tagen ohne jedes Eingreifen ab. Da die Abheilung bzw. das Fernbleiben von Diarrhöen die conditio sine qua non des Gedeihens ist, wird man die Seltenheit der Diarrhöen nicht statistisch zu erweisen brauchen, sondern sich mit der Feststellung der Ernährungsresultate, wie sie sich zahlenmäßig im Gewichtsfortschritt kundgeben, begnügen dürfen.

Ohne Infektion eingeliefert wurden 11 über 1 Monat alte Kinder, 10 davon wurden mit gewöhnlichen Milchmischungen ernährt, 1 mit Eiweißmilch (berücksichtigt sind nur die Kinder mit einem mehr als 
2 Wochen dauernden Aufenthalt). Ein Ernährungsmißerfolg war in dieser Gruppe von Kindern überhaupt nicht zu verzeichnen. Der durchschnittliche wöchentliche Gewichtszuwachs betrug $160 \mathrm{~g}$.

Einen schärferen Indikator der erreichten Resultate bietet der Ernährungserfolg bei Kindern unter 1 Monat. Im ganzen wurden 33 Kinder unter 1 Monat auf den Boxenstationen länger als 4 Wochen ohne Hinzutritt einer Infektion verpflegt. Von diesen 33 machten 7, das sind $21 \%$, Schwierigkeiten bei der Aufziehung, während der weitaus größte Prozentsatz gut gedieh. 16 von den 26 gedeihenden Kindern wurden mit einfach Halbmilchzuckermischungen ernährt, in 10 Fällen wurde von vonrherein Eiweißmilch angewendet. Gedeihliche Entwicklung ist bei Emährung mit Halbmilch in unserer Anstalt und auch wohl in ander en sonst überaus selten; früher oder später pflegt der Eintritt eines Durchfalls das Ernährungsresultat zu beeinträchtigen und, wenn man ernstere Störungen hintanhalten will, einen Wechsel der Ernährungsmethode zu erfordern (Frauenmilch oder Eiweißmilch). Es darf deshalb das günstige Ernährungsresultat bei Verabreichung von Halbmilchmischungen als besonders bedeutsam bezeichnet werden. Im Durchschnitt aller Fälle wurde ein wöchentlicher Gewichtszuwachs von $124 \mathrm{~g}$ erzielt, ein wohl zufriedenstellendes Resultat.

In 7 Fällen mußte, wie vorher erwähnt, infolge eingetretener Durchfälle von der üblichen künstlichen Ernährung abgegangen und Eiweißmilch angewendet werden. Dabei fand in allen 7 Fällen völlige Erholung statt.

Vollends erwiesen wird die überragende Bedeutung der Ausschaltung der Infektion für den Ernährungserfolg im Spital, wenn es gelingt, auch die jüngsten Säuglinge unter geringeren Schwierigkeiten als bisher mit den üblichen Methoden aufzuziehen. Von den 33 Kindern unter 1 Monat waren 24 unter 14 Tage alt, also im landläufigen Sinne Neugeborene. Nur 6 mal, d. i. in $25 \%$ der Fälle, war ein Wechsel in der Ernährungsart erforderlich (von den 7 Ernährungsmißerfolgen. bei Säuglingen unter 1 Monat betrafen also 6 - Kinder der ersten 14 Lebenstage).

9 Neugeborene gediehen bei einer Ernährung mit Halbmilch und $5 \%$ Nährmaltose (Fig. 12, 13, 14, 15 u. 16), einer Mischung, die bei Neugeborenen im Milieu unseres Spitals erfahrungsgemä $B$ fast ausnahmslos zu Mißerfolgen führt. Als Zeichen des guten Fortschritts dieser Kinder sei die durchschnittliche wöchentliche Gewichtszunahme. von $124 \mathrm{~g}$ angeführt. Aber nicht nur bezüglich der Gewichtszunahme, 

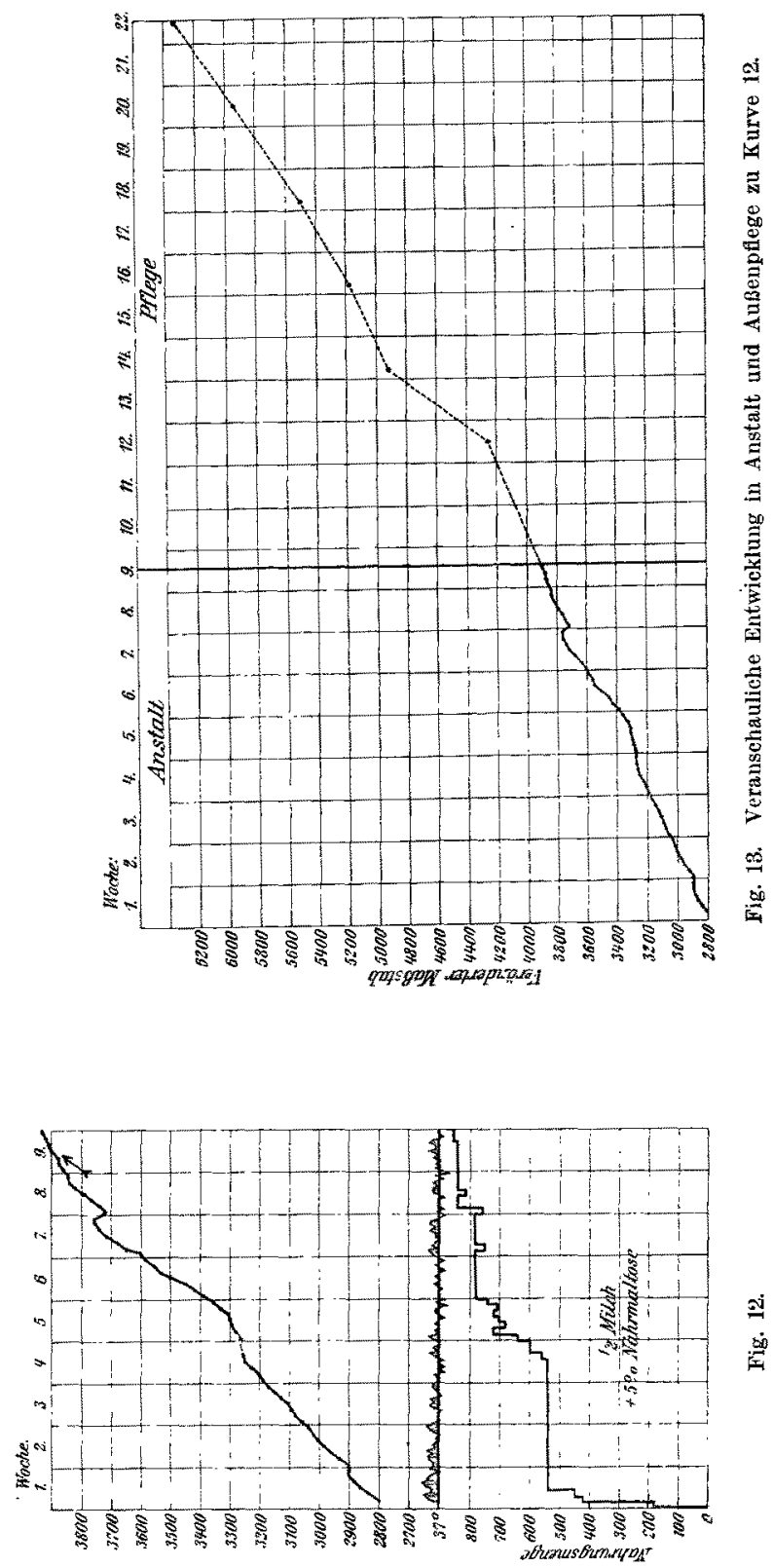


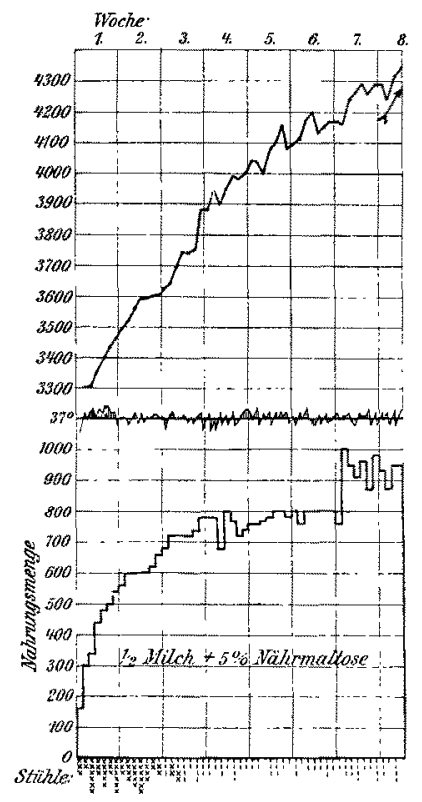

Fig. 14.

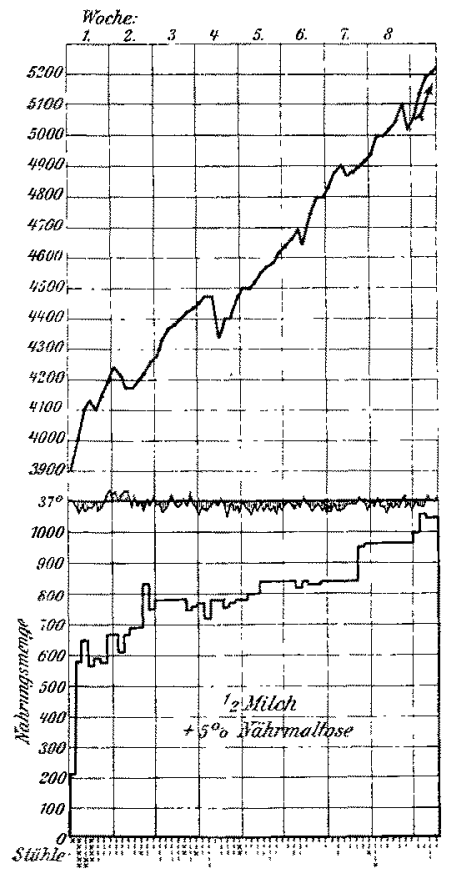

Fig. 16.

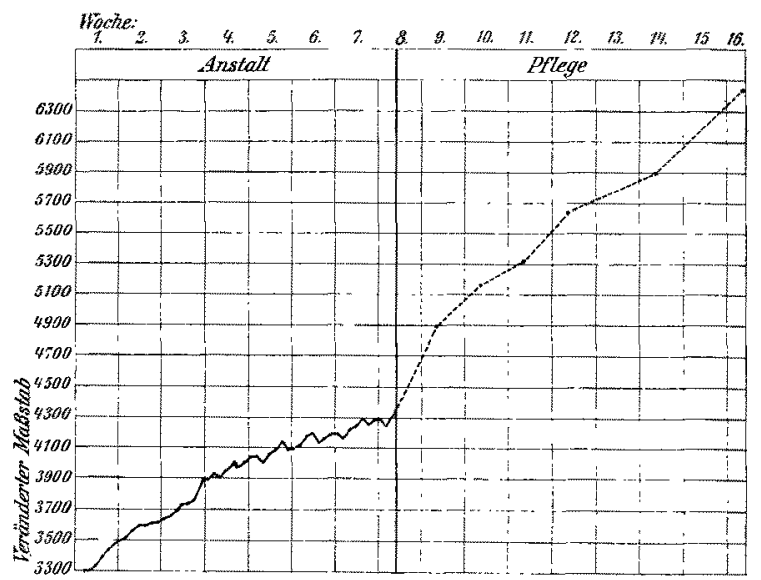

Fig. 15. Veranschaulicht die Entwicklung in Anstalt und AuBenpflege zu Kurve 14.

auch in den sonstigen Qualitäten der Gesundheit standen diese Kinder hinter berechtigten Anforderungen nicht zurück.

Gleich günstig war das Resultat der restierenden 9 Fälle, die gleich von der Aufnahme ab mit Eiweißmilch ernährt wurden und im Durchschnitt pro Woche $132 \mathrm{~g}$ zunahmen.

Im Gegensatz zu diesen erfreulichen Resultaten bei infektionsfreicn Kindern stehen die ungünstigen Resultate bei Kindern, die mit Infektionen grippaler Art auf die Station aufgenommen wurden. Von $\mathbf{1 7}$ jungen Kindern im ersten Lebensmonat dieser Gruppe schlug der Ernährungserfolg bei Darreichung üblicher Mischungen 11 mal fehl, 2 Kinder wurden von Anbeginn mit Eiweiß-

(Fortsetzung siehe s. 264.) 
Ernährungserfolge bei jungen Kindern ohne Infektionen

\begin{tabular}{|c|c|c|c|c|c|c|}
\hline & Name & $\begin{array}{c}\text { Alter } \\
\text { und Gewicht }\end{array}$ & $\begin{array}{c}\text { Dauer } \\
\text { W. } \\
\end{array}$ & $\begin{array}{c}\text { Zunahme } \\
\mathrm{g}\end{array}$ & $\begin{array}{c}\text { Pro Woche } \\
\mathrm{g}\end{array}$ & Ernährungsart \\
\hline 1 & F. Blmo & $\begin{array}{c}1 \text { Monat } \\
3780 \mathrm{~g}\end{array}$ & 10 & 1200 & 120 & ${ }_{i n} \mathrm{M}$. \\
\hline 2 & R. Jurkau & 1 Monat & 6 & 850 & 140 & ${ }^{1}: 3$ \\
\hline 3 & E. Früsch & 9 Tage & 10 & 1000 & 100 & $1_{i 2 \pi}$ \\
\hline 4 & H. Lose & $\begin{array}{l}1 \text { Monat } \\
3450 \mathrm{~g}\end{array}$ & $3^{1}: 2$ & 300 & $10 \mathrm{~J}$ & $\because "$ \\
\hline $\bar{y}$ & K. Nuller & $\begin{array}{l}7 \text { Tage } \\
2720 \mathrm{~g}\end{array}$ & 10 & 1150 & 115 & $\therefore 2$ \\
\hline 6 & E. Baganz & $\begin{array}{l}14 \text { Tage } \\
3260 \mathrm{~g}\end{array}$ & 9 & 1000 & 110 & 12 \\
\hline 7 & H. Jopsky & $\begin{array}{l}3 \text { Wochen } \\
3580 \mathrm{~g}\end{array}$ & 9 & 900 & 100 & $\begin{array}{l}4 \text { W. E.-M. } \\
\text { (ann } 1 / 2 \text { M. }\end{array}$ \\
\hline 8 & Dombrowsky & $\begin{array}{c}1 \pm \text { Tage } \\
3250 \mathrm{~g}\end{array}$ & 9 & 1500 & 160 & $\begin{array}{l}8 \mathrm{~W} . \mathrm{E} . \mathrm{M} \\
\text { dann } \\
\mathrm{M}\end{array}$ \\
\hline 9 & F. Tanks & $\begin{array}{l}16 \text { Tage } \\
3200 \mathrm{~g}\end{array}$ & 5 & 700 & 140 & E. M. \\
\hline 10 & Ch. Graffert & $\begin{array}{l}9 \text { Tage } \\
3000 \mathrm{~g}\end{array}$ & 5 & 800 & 160 & ${ }^{1 / 2} \mathrm{M}$. \\
\hline 11 & K. Stroschein & $\begin{array}{l}9 \text { Tage } \\
2 \pm 80 \mathrm{~g}\end{array}$ & 11 & 1120 & 110 & 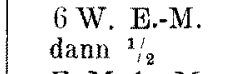 \\
\hline 12 & Kostas & $\begin{array}{l}\text { 14 Tage } \\
3180 \mathrm{~g}\end{array}$ & 5 & 620 & 120 & E.-M. ${ }^{1}{ }_{2} M$ \\
\hline 13 & Kanatschke & $\begin{array}{l}6 \text { Tage } \\
2900 \mathrm{~g}\end{array}$ & 7 & 900 & 130 & E.-M. ${ }^{1} \mathrm{M}$. \\
\hline 14 & Kazuba & $\begin{array}{l}3 \text { Wochen } \\
3000 \mathrm{~g}\end{array}$ & 9 & 950 & 95 & $\left.1 / 2 \mathrm{M} .{ }^{1}\right)$ \\
\hline 15 & E. Kranzow & $\begin{array}{l}14 \text { Tare } \\
3520 \mathrm{~g}\end{array}$ & 10 & 1600 & 160 & $\begin{array}{l}5 \mathrm{~W} . \mathrm{E}_{.}-\mathrm{M} \\
\operatorname{dann} \overline{1 / 2} \mathrm{M}\end{array}$ \\
\hline 16 & E. NIros & $\begin{array}{l}10 \text { Tage } \\
3450 \mathrm{~g}\end{array}$ & 9 & 900 & 100 & I M. \\
\hline $1 \overline{7}$ & $\begin{array}{l}\text { H. Meirich } \\
\text { geb. Zwilling }\end{array}$ & $\begin{array}{l}14 \text { Tage } \\
2100 \mathrm{~g}\end{array}$ & 11 & 1400 & 130 & $\begin{array}{c}\text { dann Brust olin, } \\
\text { zwingendeIndication }\end{array}$ \\
\hline 18 & Erna Hein & $\begin{array}{l}14 \text { Tage } \\
3650 \mathrm{~g}\end{array}$ & 9 & 900 & 100 & 1/2 M. \\
\hline 19 & A. Grega & $\begin{array}{l}3 \text { Wochen } \\
360 \mathrm{~g}\end{array}$ & 9 & 900 & 100 & a M. \\
\hline 20 & E. Rehbein & $\begin{array}{l}3 \text { Wochen } \\
+280 \mathrm{~g}\end{array}$ & 6 & 800 & 100 & $\begin{array}{l}\quad 1 / 2 \mathrm{M} \\
1 / \mathrm{F}\end{array}$ \\
\hline 21 & L. Bayer & $\begin{array}{l}10 \text { Tage } \\
3100 \mathrm{~g}\end{array}$ & 13 & 1200 & 90 & wegen Furunkulose \\
\hline $9 \cdot 9$ & G. Kogiseh & $\begin{array}{l}12 \text { Taue } \\
2730 \mathrm{~g}\end{array}$ & 9 & 1270 & 140 & $1 / 2 \mathrm{M}$. \\
\hline 23 & K. Brill & $\begin{array}{l}4 \text { Tage } \\
3240 \mathrm{~g}\end{array}$ & 8 & 1100 & $1+0$ & : M. \\
\hline 24 & T. Wink & $\begin{array}{l}10 \text { Tage } \\
3900 \mathrm{~g}\end{array}$ & 9 & 1350 & 150 & : M. \\
\hline 25 & K. Schmidt & $\begin{array}{l}11 \text { Tage } \\
2 \pm 40 \mathrm{~g}\end{array}$ & 12 & 1860 & 150 & dam. ${ }_{2}=-M$ \\
\hline 26 & E. Huher & $\begin{array}{l}8 \text { Tage } \\
2900 \mathrm{~g}\end{array}$ & 12 & 1700 & 140 & $\begin{array}{l}8 \mathrm{~W} . \text { E.-M. } \\
\text { dann } 1 / 2 \mathrm{M} .\end{array}$ \\
\hline
\end{tabular}


Ernährungsmißerfolge bei jungen Kindern ohne Infektionen.

\begin{tabular}{|c|c|c|c|c|}
\hline Nr. & Name & $\begin{array}{c}\text { Alter } \\
\text { und Gewicht }\end{array}$ & $\begin{array}{l}\text { Dauer } \\
\text { w. }\end{array}$ & Ernährungsart \\
\hline 1 & M. Frühloff & $\begin{array}{l}7 \mathrm{Tg} \\
3000 \mathrm{~g}\end{array}$ & $4 \mathrm{~W}$. & $\begin{array}{l}1 / 2 \text { M., } 1^{1 / 2} \text { W., sofort Durchfall, } \\
200 \mathrm{~g} \text { Abnahme, mit E.-M. Zunahme. }\end{array}$ \\
\hline 2 & E. Bär & $\begin{array}{l}13 \mathrm{Tg} . \\
3250 \mathrm{~g}\end{array}$ & $11 \mathrm{M}$. & $\begin{array}{l}\text { Zuerst } 5 \text { Wochen bei B.-M. Dyspep- } \\
\text { sie. } 200 \mathrm{~g} \text { Abnahme, dan bei } \\
1 / \mathrm{g} \text { M. mit Käse in } 6 \text { Wochen } \\
900 \mathrm{~g} \text { Zunahme. }\end{array}$ \\
\hline 3 & E. Slominski & $\begin{array}{l}14 \mathrm{Tg} . \\
2600 \mathrm{~g}\end{array}$ & $12 \mathrm{~W}$. & $\begin{array}{l}\text { Bei } 1 / \text { M. Dyspepsie } 1 \text { Woche, dann } \\
\text { E.-M., später } 1 / 2 \mathrm{M} \text {. Zunahme } \\
1300 \mathrm{~g} \text {, pro Woche } 110 \mathrm{~g} \text {. }\end{array}$ \\
\hline 4 & Nerczon & $\begin{array}{l}7 \mathrm{Tg} \\
2800 \mathrm{~g}\end{array}$ & $8 \mathrm{~W}$. & $\begin{array}{l}\text { Zuerst 1/2 M. Abfall, dann E.-M. } \\
\text { Zunahme. In } 6 \text { Wochen } 400 \mathrm{~g} \text { Zu- } \\
\text { nahme. } \\
2 \text { Wochen bei } 1 / 2 \text { M. Absceß }\end{array}$ \\
\hline 5 & H. Schlegel & $\begin{array}{l}9 \mathrm{Tg} . \\
3340 \mathrm{~g}\end{array}$ & $8 \mathrm{~W}$ & $\begin{array}{l}\text { Dyspepsie, nachher E.-M. Gutes Ge- } \\
\text { deihen. } 1 \mathrm{~kg} \text { Zunahme in } 5 \text { Wochen. } \\
\text { Bei } 1_{2} \text { M. Dyspepsie } 150 \mathrm{~g} \text { Abnahme }\end{array}$ \\
\hline 6 & G. Krause & $\begin{array}{l}3 W \\
3650 \mathrm{~g}\end{array}$ & $9 \mathrm{~W}$ & $\begin{array}{l}\text { in } 2 \text { Wochen. Dann E.-M. in } \\
7 \text { Wochen } 1400 \mathrm{~g} \text { Zunahme. } \\
4 \text { Wochen Iang bei } 1 / 2 \text { M. Nicht hei- }\end{array}$ \\
\hline 7 & Schubert & $\begin{array}{l}10 \mathrm{Tg} . \\
3000 \mathrm{~g}\end{array}$ & $13 \mathrm{~W}$. & $\begin{array}{l}\text { lende Dyspepsie ohne Zunahme. } \\
\text { Dann E.-M. Gute Zunahme in } \\
8 \text { Wochen, } 110 \mathrm{~g} \text { g. }\end{array}$ \\
\hline
\end{tabular}

Altere Kinder, obne Infektion.

\begin{tabular}{|c|c|c|c|c|c|}
\hline Nr. & Name & Alter & & $\begin{array}{l}\text { Gewichtszu- } \\
\text { nahme pro } \\
\text { Woche }\end{array}$ & Eruährungsart \\
\hline 1 & R. Rogall & $2^{1}{ }_{2}$ Mon. & 5 & 140 & ${ }_{1}^{1}$ Mileh \\
\hline 2 & F. Rösbery & 2 & 5 & 160 & 1. \\
\hline 3 & D. Kaffka & & 5 & 140 & $1_{2}$, \\
\hline 4 & M. Weissenbach & & 3 & 210 & $2_{3}$ \\
\hline 5 & F. Patzlaff & $2 n$ & 2 & 120 & $\mathbf{1}_{2}$ \\
\hline 6 & Kurt Lehmann & $1_{2}^{1}$ & 4 & 200 & E.M. ${ }^{2}{ }_{3}$, \\
\hline 7 & H. Bosse & & 5 & 140 & 1 \\
\hline 8 & E. Wernike & 3 & 4 & 150 & Malzsuppe \\
\hline 9 & H. Grigel & & 4 & 150 & $1 / 2$ Milch \\
\hline 10 & K. Bormann & 6 Wochen & 5 & 160 & $1: 2$ \\
\hline 11 & M. Jäckel. & 3 Mon. & 3 & 200 & $1_{2}$ \\
\hline
\end{tabular}


Junge Kinder, mit Infektionen eingeliefert.

\begin{tabular}{|c|c|c|c|c|c|c|c|}
\hline $\mathrm{Nr}$. & Name & $\begin{array}{c}\text { Alter } \\
\text { und } \\
\text { Gewicht }\end{array}$ & \begin{tabular}{|} 
Dauer \\
Wochen \\
\end{tabular} & $\begin{array}{c}\text { Gew:- } \\
\text { Zul- } \\
\text { nahme } \\
\mathrm{g} \\
---\end{array}$ & $\begin{array}{c}\text { Pro } \\
\text { Woche } \\
\mathrm{g}\end{array}$ & $\begin{array}{c}\text { Mißerfolg } \\
\text { bei }\end{array}$ & $\begin{array}{c}\text { Erfolg } \\
\text { bei }\end{array}$ \\
\hline 1 & $\begin{array}{l}\text { H. Drinkmann } \\
\text { Grippe }\end{array}$ & $\begin{array}{l}11 \text { Tage } \\
3070 \mathrm{~g}\end{array}$ & .9 & 1000 & 110 & : M. & E. - M. \\
\hline 2 & $\begin{array}{l}\text { L. Brangseh } \\
\text { Grippe }\end{array}$ & $\begin{array}{l}8 \text { Tage } \\
3300 \mathrm{~g}\end{array}$ & 2 & -600 & - & ${ }^{1}, \mathrm{M}$. & $\dagger$ \\
\hline 3 & $\begin{array}{l}\text { E. Lanzrat } \\
\text { Grippe } \\
\text { schwere parent. } \\
\text { Ernährungs- } \\
\text { Störung, 1 kg } \\
\text { Alnahme in den } \\
\text { ersten } 3 \text { Woch., } \\
\text { dabei stärkeres } \\
\text { Auftr. v. Husten }\end{array}$ & $\begin{array}{c}1 \text { Monat } \\
4000 \mathrm{~g}\end{array}$ & 9 & 200 & 20 & $1_{i 2}^{\prime} \mathrm{M}$. & E.-M. \\
\hline 4 & $\begin{array}{l}\text { H. Nue } \\
\text { Grippe }\end{array}$ & $\begin{array}{l}1 \text { Monat } \\
3300 \mathrm{~g}\end{array}$ & 3 & 100 & 33 & - & ${ }_{12} \mathrm{M}$. \\
\hline$\overline{5}$ & $\begin{array}{l}\text { II. Buschat } \\
\text { Grippe }\end{array}$ & $\begin{array}{l}3 \text { Woch. } \\
3600 \mathrm{~g}\end{array}$ & 4 & -400 & -100 & $\begin{array}{l}\text { Zunächst } \\
\text { Abnahme }\end{array}$ & $E_{3}-M$ \\
\hline 6 & $\begin{array}{l}\text { A. Bettin } \\
\text { Grippe }\end{array}$ & $\begin{array}{l}1 \text { Monat } \\
3800 \mathrm{~g}\end{array}$ & 9 & - & - & $\begin{array}{l}\text { zuerst B.-M., da }- \\
\text { bei Intoxikation } \\
\text { mit } 400 \mathrm{~g} \\
\text { Abnahme in } \\
3 \text { Wochen }\end{array}$ & $\begin{array}{l}\text { E.-M. mit } \\
750 \mathrm{~g} \mathrm{Zu}- \\
\text { nahme in } \\
4 \text { Wochen }\end{array}$ \\
\hline $\bar{\imath}$ & $\begin{array}{l}\text { A. Grein } \\
\text { Grippe }\end{array}$ & $\begin{array}{l}3 \text { Woch. } \\
3700 \mathrm{~g}\end{array}$ & 6 & 650 & 110 & - & $\begin{array}{l}1: \mathrm{M} \\
\mathrm{M} \cdot \mathrm{S} \text {. }\end{array}$ \\
\hline 8 & $\begin{array}{l}\text { WT. Reiß } \\
\text { Grippe }\end{array}$ & $\begin{array}{l}2 \text { Woch. } \\
4000 \mathrm{~g}\end{array}$ & 6 & 一 & - & $\left|\begin{array}{c}\text { parent. Störung } \\
\text { mit } 600 \mathrm{~g}-\text { in } \\
3 \text { Wochen, } \\
\text { dann } 3 \text { Wochen } \\
600 \mathrm{~g}+\end{array}\right|$ & E.-M. \\
\hline 9 & $\begin{array}{l}\text { Falmmann } \\
\text { Pemphigus }\end{array}$ & $\begin{array}{l}\text { 10Tage } \\
2800 \mathrm{~g}\end{array}$ & 15 & 1150 & 80 & $\begin{array}{c}\text { zuerst } 4 \text { Wochen } \\
1 / 2 \text { M., dann B.- } \\
\text { M., dabei Dys- } \\
\text { pepsie, dann } \\
\text { E.-M. }\end{array}$ & E.-M. \\
\hline 10 & $\begin{array}{c}\text { Harpenow, } \\
\text { Grippe,Pyelitis, } \\
\text { o Woch. Husten }\end{array}$ & 1 Monat & 7 & 450 & 65 & $1 / 2 \mathrm{M}$. & E.-M. \\
\hline 11 & $\begin{array}{l}\text { A. Meyer, } \\
\text { Husten }\end{array}$ & 12 Tage & 10 & 950 & 95 & - & $12 \mathrm{M}$. \\
\hline
\end{tabular}


(Fortsetzung).

\begin{tabular}{|c|c|c|c|c|c|c|c|}
\hline Nr. & Name & $\begin{array}{c}\text { Aiter } \\
\text { und } \\
\text { Gewicht }\end{array}$ & $\begin{array}{c}\text { Dauer } \\
\text { Wochen }\end{array}$ & $\begin{array}{c}\text { Gew.- } \\
\text { Zü- } \\
\text { natime } \\
g\end{array}$ & $\begin{array}{c}\text { Pro } \\
\text { Woche } \\
\mathrm{g}\end{array}$ & $\begin{array}{c}\text { Mrikeriolg } \\
\text { bei }\end{array}$ & $\begin{array}{l}\text { Errolg } \\
\text { bei }\end{array}$ \\
\hline 12 & $\begin{array}{c}\text { K. Lempke } \\
\text { Schnupfen, } \\
\text { Husten, } 7 \text { Woch. } \\
\text { Ohrenlaufen, } \\
\text { lange Zeit Tem- } \\
\text { peraturzacken, } \\
\text { häufig. dünnere } \\
\text { Stuhle }\end{array}$ & 3 Woch. & 10 & 1400 & 140 & - & $1_{2} \mathrm{M}$. \\
\hline 13 & $\begin{array}{c}\text { E. Hickmann } \\
\text { Grippe, Dys- } \\
\text { pepsie bei }{ }^{1} / 2 \mathrm{M} ., \\
\text { nach } 4 \text { Wochen } \\
\text { Ohrenlaufen, } \\
\text { wiederholte } \\
\text { Bronchitis }\end{array}$ & 1 Monat & 6 & -140 & -40 & $\begin{array}{c}\text { Mißerfolg bei } \\
{ }_{1}, \mathrm{M} .\end{array}$ & E.-M. \\
\hline 14 & $\begin{array}{l}\text { R. Rutner } \\
\text { Grippe }\end{array}$ & 12Tage & 9 & 500 & 55 & $\begin{array}{c}\text { Mißerfolg bei } \\
1 / 2 \mathrm{M}\end{array}$ & $\begin{array}{c}\text { gute Zunahme } \\
\text { bei E.-M. }\end{array}$ \\
\hline 15 & $\begin{array}{l}\text { E. Hornig } \\
\text { Grippe }\end{array}$ & 1 Monat & 9 & 650 & 70 & $1 / 2 \mathrm{M}$. & $\begin{array}{l}\text { E.-M. } \\
:=\mathrm{M} .\end{array}$ \\
\hline 16 & $\begin{array}{c}\text { W. Buge } \\
\text { Grippe, } 7 \text { Woch. } \\
\text { Husten }\end{array}$ & 9 Tage & 9 & - & - & $\begin{array}{c}1 / 2 \text { M.Dyspepsie, } \\
\text { dann E.-M. }\end{array}$ & E.-M. \\
\hline 17 & $\begin{array}{l}\text { W. Schmidt } \\
\text { lange Zeit } \\
\text { schwer. Husten }\end{array}$ & 10 Tage & 7 & 500 & 70 & $\begin{array}{l}\text { bei } 1 / 2 \text { M. dau- } \\
\text { ernd Dyspepsie }\end{array}$ & E.-M. \\
\hline 18 & $\begin{array}{c}\text { W. Krahn } \\
\text { Grippe }\end{array}$ & $\begin{array}{l}11 \text { Tage } \\
3180 \mathrm{~g}\end{array}$ & 15 & 1 & 60 & $\begin{array}{c}\text { die ersten } \\
\text { Z.-W. b. } 1 / 2 \text { M. } \\
\text { und B.-M. } \\
\text { Dyspepsie }\end{array}$ & E. M. \\
\hline
\end{tabular}

milch ernährt und nur 4 mal wurde mit Halbmilchmischung befriedigendes Gedeihen erzielt. Ein Kind starb an parenteraler Störung. Während also von 23 Kindern des gleichen Lebensabschnittes bei Infektionsfreiheit nur 7 Mißerfolge bei Ernährung mit gewöhnlicher Milchmischung eintraten, gleich $30 \%$, stieg diese Zahl der Mißerfolge 
bei den von vornherein infizierten Kindern in 15 Fällen auf 11, gleich $73 \%$ der Fälle.

Selbstrerständlich wurde auch das gesamte Ernährungsresultat in cler Gruppe der Infizierten sehr beeinträchtigt, der Gewichtsfortschritt blieb wesentlich hinter dem der Infeltionsfreien zurück und betrug im wöchentlichen Durchschnitt $45 \mathrm{~g}$ gegen $124 \mathrm{~g}$. (Bei der Berechnung wurde die Gewichtsabnahme bei der einen tödlich endenden Erkrankung nicht berücksichtigt.)

Die Ernährungsresultate auf den Boxenstationen haben also durch den Ausschluß der Infektion eine wesentliche Verbesserung erfahren, die sich am sinnfälligsten bei der Ernährung junger Kinder und Neugeborener zeigte und in der Einschränkung akuter Verdauungsstörungen bestand.

Ich habe versucht, die erreichten Verbesserungen mit nüchternen Zahlen auszudrücken, die freilich nur ein unzulängliches Bild des Erreichten geben. Wer den Unterschied in den Pflegeresultaten zwischen den Boxenstationen und den anderen Stationen unseres Hauses mit uns beobachtet hat, der muß die Einrichtung als Fortschritt auf dem Wege der Sanierung des Säuglingsspitals, den uns zuerst Heubner gewiesen hat, begrüßen. Wenn auch die Einrichtungen noch verbesserungshedürftig sind, im Prinzip liegt die Einschränkung der Infektion durch die Verhinderung der Luftübertragung und die dadurch zu erzielende Melioration der Ernährungsresultate nicht mehr außerbalb des Bereiches der Möglichkeit.

Berlin W. 35, Genthinerstr. $19 \mathrm{I}$. 